\title{
Ship generated mini-tsunamis
}

\author{
John Grue $^{1} \dagger$ \\ ${ }^{1}$ Mechanics Division, Department of Mathematics, University of Oslo, Oslo, Norway
}

(Received xx; revised xx; accepted xx)

Very long waves are generated when a ship moves across an appreciable depth change $\Delta h$ comparable to the average and relatively shallow water depth $h$ at the location, with $\Delta h / h \simeq 1$. The phenomenon is new and the waves are recently observed in the Oslofjord in Norway. The $0.5-1 \mathrm{~km}$ long waves, extending across the $2-3 \mathrm{~km}$ wide fjord, are observed as run-ups and run-downs along the shore, of periods of 30-60 seconds, where a wave height up to $1.4 \mathrm{~m}$ has been measured. The waves travelling with the shallow water speed, found ahead of the ships moving at subcritical depth Froude number, behave like a mini-tsunami. A qualitative explanation of the linear generation mechanism is provided by an asymptotic analysis, valid for $\Delta h / h<<1$ and long waves, expressing the generation in terms of a pressure impulse at the depth change. Complementary fully dispersive calculations for $\Delta h / h \simeq 1$ document symmetries of the waves at positive or negative $\Delta h$. The wave height grows with the ship speed $U$ according to $U^{n}$ with $n$ in the range $3-4$, for $\Delta h / h \simeq 1$, while the growth in $U$ is only very weak for $\Delta h / h<<1$ (the asymptotics). Calculations show good agreement with observations.

Key words: Waves/Free-surface Flows, Channel flow, Topographic Effects

\section{Introduction}

Very long waves travelling upstream of ships are a recent observation in the Oslofjord, an inlet in Norway extending southward from Oslo into the Skagerrak. The phenomenon is new and the generation process is described here for the first time. The waves are formed when the new very large and relatively fast cruiseferries, which are conventional ships, travel across substantial depth changes, $\Delta h$, where $\Delta h$ typically is comparable to the average and relatively shallow water depth, $h$, at the location, i.e. $\Delta h / h \sim 1$. The generation mechanism is linear. The waves running ahead of the ships propagate with the shallow water speed. The periods in the range 30-60 seconds are observed at the shore where shorter waves travel behind the main waves. The wave length is $0.5-1 \mathrm{~km}$, and crests extend across the $2-3 \mathrm{~km}$ wide fjord. The ship speed $U$ is subcritical with a depth Froude number $U / \sqrt{g h}$ in the range $0.4-0.7$, where $g$ is acceleration of gravity. The Froude number based on the ship length $l$ is in the range $F r_{l}=U / \sqrt{g l} \sim 0.17-0.24$.

Documentation of observed wave heights, wave-induced currents and the consequence regarding erosion is provided in Appendix A.1. A wave height of $1 \mathrm{~m}$ at specific locations along the shore is typical, and a wave height of up to $1.4 \mathrm{~m}$ has been measured. The wave-induced currents of up to about $1 \mathrm{~ms}^{-1}$, particularly the long lasting run-outs of approximately 15-30 seconds (half wave period), contribute to a significant, new kind of erosion. This is according to reports from a group of landlords in the village of Flaskebekk, at the Oslofjord, where small traditional bath houses on the shore as well as piers - a cultural landscape dating back to the 1890s - are built on unconsolidated sediments with some fractions of rock. They are slowly sliding towards the sea because of the new

$\dagger$ Email address for correspondence: johng@math.uio.no 
wave phenomenon and erosion. Marinas experience damaged as well. According to the landlords the waves appeared in 2004 with the introduction of Fantasy, a cruiseferry of Color Line, which together with Magic are the world's largest of their kind. Their displaced volume is 46 per cent larger than Perl Seaways of DFDS which started operating in the Oslofjord in 2001 and 78 per cent larger than Crown Seaways of DFDS, introduced in 1994, where the ship characteristics are given in table 4 in Appendix A.1. The ships of Color Line are cruising at a higher speed (up to 23 knots has been observed) compared to DFDS (16-17 knots), at the locations where the waves are generated.

Studying a variant of the present problem, Sibul, Webster and Wehausen (1979) performed force measurements in a towing tank of a ship moving over a rectangular box, obtaining a force response localized in time and a subsequent slowly decaying almost periodic signal. However, both the analysis and results were rather fragmented, with no firm conclusions. Other descriptions of the upstream waves we study seem not to exist. The mechanism investigated here differs fundamentally from the transcritical upstream waves due to a ship in water of constant depth where the upstream waves are caused by nonlinearity. Early experiments of the critical range by Constantine (1960) showed that a quantity of fluid continuously was piled up ahead of the ship in the form of a bore. Conversely, the flow conditions in the subcritical and supercritical ranges were steady. Upstream, transcritical generation of solitons in two and three dimensions have been much explored, see e.g., Cole (1985), Ertekin, Webster and Wehausen (1986), Wu (1987) and Pedersen (1988).

The downstream wave wake of fast ships with a speed in the range of 30-35 knots and Froude number based on the ship length exceeding $F r_{l}>0.4$ (e.g. Torsvik, 2009) has received attention in a number of publications. A set of experimental observations of an oblique solitary wave packet in the Kelvin wake of a fast ship, at sea, at $F r_{l}=0.49$, in deep water, was analysed by Brown et al. (1989). Combined with the shallow water effect the fast ships may produce destructive waves at the shore where a wave heigth of up to 5 $\mathrm{m}$ has been reported (Hamer, 1999). Measurements of the wave wakes of the fast ships, in the Bay of Tallinn, show three distinct groups of waves where the longest and fastest waves, of period in the range of 10-15 seconds and height of $1 \mathrm{~m}$, dominate over the wind waves, see Soomere (2005). Moreover, unusually high hydrodynamic loads in the deeper parts of the near shore are caused by the longer wake waves, see particularly $\S 4.4$ in Soomere (2009). The dynamical similarity of the leading $200 \mathrm{~m}$ long diverging wave in the wave wake of a fast ship, at a water depth of $20 \mathrm{~m}$, including the run-up at the beach, are compared to seismic and slide generated tsunamis in Didenkulova, Pelinovsky and Soomere (2011). The wave wake close to the critical depth Froude number, in a channel with a deeper trench and shallower depth at the sides, analysed by Torsvik, Pedersen and Dysthe (2009), exhibited a wave amplification due to the downstream wave packet. Their nonlinear calculations obtained upstream solitary wave generation similarly as described in the previous paragraph. The wave resistance in shallow water in subcritical and supercritical conditions shows a variation due to a varying depth ( Wu and Chen, 2003).

Although at a small length scale, the waves in consideration share many similarities of tsunamis caused by tectonics (e.g. Glimsdal et al., 2006), submarine slides (e.g. Harbitz, 1992), underwater vulcano eruptions or explosions, the latter with reported wavelengths in the range 0.1-1 km (e.g. Pelinovsky, 2006). The present waves are generated localized in time and space, by a mechanism of impulsive type, which is confirmed by the analysis and calculations in $\S \S 4,5$ below. The long waves in the Oslofjord propagating with the shallow water speed are hard to detect in the middle of the fjord. Rather they are observed by the slow run-ups and run-downs in the bays and harbors along the shore where people 
have been using the term tsunami - harbour wave - according to the Japanese origin. We have found it tempting to characterize the present upstream ship generated waves as a mini-tsunami.

We provide a mathematical and numerical analysis as well as an explanation and calculation of the new phenomenon, investigating of the upstream wave generation by the ships moving at subcritical speed across the depth changes. The paper is organized as follows. Observations are described in $\S 2$. The mathematical formulation based on linear potential theory, integral equations and Fourier transform for the solution of the Laplace equation is given in $\S 3$. An asymptotic analysis valid for small wavenumber and $\Delta h / h<<1$ is provided in $\S 4$. Fully dispersive calculations at a positive or negative depth change with $\Delta h / h \sim 1$ in a relatively narrow channel are given in $\S 5$. Results for a wide channel are given in $\S 6$. Conclusions are presented in $\S 7$.

\section{Observations}

The wave periods are observed at the shore line. Waves of shorter period succeed the main waves. The onsets of the inflows and outflows in a particular harbor in Flaskebekk, on the eastern side of the Oslofjord, were by the author recorded on 17 February 2016 using a stop watch. The wave periods due to the two ships DFDS Pearl Seaways and Color Fantasy cruising by Flaskebekk, observation position 1, the first right after 9 a.m., at a speed of $8.6 \mathrm{~ms}^{-1}$, the second right before 9:30 a.m., at a speed of $10.3 \mathrm{~ms}^{-1}$, were approximately 34 seconds for both ships, see table 1 . A corresponding wavelength of approximately $830 \mathrm{~m}$ can be estimated by multiplying the period by the shallow water speed in the fjord. Note that the first inflow-to-inflow period of 26 seconds due the first slower and somewhat smaller ship may not be accurate, since the wave amplitude was rather small. A third wave period of 13 seconds due to the second, faster and larger ship was not generated by the slower one. A typical wave height of $1 \mathrm{~m}$ is documented in this harbor, and a record height of $1.4 \mathrm{~m}$ has been measured by the owner, see the further documentation in Appendix A.1.

A photo of the outflow in the harbor due to the dominant depression wave is shown in figure 1. The ship Color Magic causing the harbor wave is seen to the right in the picture. The wave is generated at a position in the fjord located right behind Ildjernsflu lighthouse which is seen in the picture above the pier, to the right of the white bath house. The harbor shown in figure 1 (position 1) and Ildjernsflu lighthouse are indicated on the seachart shown in figure $2 \mathrm{a}$, available at www.oslofjorden.com $/ \mathrm{map} /$.

The upstream ship waves extending across the $2-3 \mathrm{~km}$ wide fjord are observed along both shores. In a small bay at Snarøya on the north-western shore of the fjord, referred to as observation position 2 , the wave periods recorded by the author were close to a minute, where the observed ship, Color Magic, was crusing at a speed of $7.8 \mathrm{~ms}^{-1}$, see table 1 . The first period of $T=57$ seconds corresponds to a wavelength of approximately 900 m. (On the day of observation, 7 January 2016, the Norwegian Coastal Administration performed blasting of 24 shallow rocks in the Inner Oslofjord, and the ship travelled at a reduced speed.)

At Askholmene islands, located midways in the fjord north of the village of Drøbak, observation position 3, the southward track is located west of the islands, see the seachart in figure $2 \mathrm{~b}$. A video recording of the upstream waves at this position due to Color Magic is available at https://www.youtube.com/watch? $\mathrm{v}=42 \mathrm{Ctdk} 9 \mathrm{kpyg}$. The video shows that the very first motion in the bay is a weak outflow. This means that the leading upstream wave caused by the ship moving along a depth that is reduced, is a weak wave of depression. Periods of the dominant wave train obtained from this video show $T=32 \pm 3$ 
Fantasy, $10.3 \mathrm{~ms}^{-1}$ Pearl Seaways, $8.6 \mathrm{~ms}^{-1}$ Magic, $U=7.8 \mathrm{~ms}^{-1}$ Magic, $U=10 \mathrm{~ms}^{-1}$ $\begin{array}{llll}\text { Position } 1 & \text { Position } 1 & \text { Position } 2 & \text { Position } 3\end{array}$

\begin{tabular}{|c|c|c|c|c|c|c|c|c|}
\hline wave no. & in-in & out-out & in-in & out-out & in-in & out-out & in-in & out-out \\
\hline 1 & $34 \mathrm{~s}$ & $36 \mathrm{~s}$ & $26 \mathrm{~s}$ & $34 \mathrm{~s}$ & $58 \mathrm{~s}$ & $57 \mathrm{~s}$ & $35 \mathrm{~s}$ & $30 \mathrm{~s}$ \\
\hline 2 & $32 \mathrm{~s}$ & - & $34 \mathrm{~s}$ & - & $49 \mathrm{~s}$ & $40 \mathrm{~s}$ & $29 \mathrm{~s}$ & $34 \mathrm{~s}$ \\
\hline 3 & $13 \mathrm{~s}$ & - & - & - & $64 \mathrm{~s}$ & - & $46 \mathrm{~s}$ & $36 \mathrm{~s}$ \\
\hline
\end{tabular}

TABLE 1. Wave periods between two consecutive inflows (in-in) and outflows (out-out) in harbors or small bays due to three different ships observed at three different positions.

seconds for the leading two waves (table 1) and correspond to a wavelength of about 550 $\mathrm{m}$.

The waves are generated when the ships travel across pronounced depth changes. Let $h_{A}$ denote the water depth along the ship's track before the depth change, $h_{B}$ the typical depth in the shallow region and $h_{C}$ depth after the shallow region. The wave generation is observed for a depth change $\Delta h=h_{A}-h_{B}$ that is comparable to the average depth $h=\frac{1}{2}\left(h_{A}+h_{B}\right)$, i.e. $\Delta h / h \simeq 1$. The ratio $2\left(h_{C}-h_{B}\right) /\left(h_{C}+h_{B}\right)$ determines the relative depth change from shallow to deep water.

The ship track at Ildjernsflu lighthouse is north/north-eastward, to the left of the pink dashed line in figure 2a. A ridge extends south/south-westward of the lighthouse where depths in meters are marked by $11 \mathrm{~m}, 16 \mathrm{~m}$ and $19 \mathrm{~m}$. From the figure we estimate: $h_{A} \approx 46 \mathrm{~m}$ and $h_{B} \approx 14 \mathrm{~m}$, giving $h=30 \mathrm{~m}$ and $\Delta h / h \simeq 1.067$. Further, an extension of $700 \mathrm{~m} \approx 23 h$ of the shallow region along the ship's track is estimated. The average depth of the fjord further north is about $60 \mathrm{~m}$ on the eastern side and about $25 \mathrm{~m}$ on the western side.

The south/south-eastward ship track at Askholmene, position 3, passes over the depths marked by $81 \mathrm{~m}, 39 \mathrm{~m}, 27 \mathrm{~m}$ in figure 2b. Note that the depth is shallower than $20 \mathrm{~m}$ next to the position marked by $39 \mathrm{~m}$. The water is only a few meters deep at each side of the track. The position of the videorecording is marked by $X$. From the figure we obtain: $h_{A} \approx 80 \mathrm{~m}$ and $h_{B} \approx 30 \mathrm{~m}$ giving $h=55 \mathrm{~m}$ and $\Delta h / h=0.909$. The distances between observation position and generation site, and the ship's track, are indicated in table 2 . Figure 2c shows the positions in the Oslofjord.

The displaced volumes of Pearl Seaways and Crown Seaways are $69 \%$ and $55 \%$, respectively, of those of Color Line, see table 4 in Appendix A.1 where also further descriptions of the observations are given.

\section{Mathematical formulation}

The analysis is three dimensional. We introduce horizontal coordinates $\mathbf{x}=\left(x_{1}, x_{2}\right)$, vertical coordinate $y$, with $y=0$ at the free surface at rest, and time $t$. The generation by the ship is modelled by a moving pressure distribution (hovercraft) of similar length, width, draught and displacement. The surface pressure is moving with constant speed $U$ along the $x_{1}$-direction where a gentle ramp up of the motion is applied. The linear potential theory accounts for the effect of the bottom variation. The waves are obtained by integrating in time the linear kinematic and dynamic boundary conditions at the free 


\begin{tabular}{|c|c|c|c|c|c|c|c|}
\hline Position & $D_{\text {source }} \mid D_{\text {track }}$ & $h_{A}$ & $h_{B}$ & $h_{C}$ & $\Delta h / h \mid$ \\
\hline 1 & $1.5 \mathrm{~km}$ & $1.2 \mathrm{~km}$ & $46 \mathrm{~m}$ & $14(11) \mathrm{m}$ & $60 \mathrm{~m}$ & 1.067 \\
2 & $2.6 \mathrm{~km}$ & $1 \mathrm{~km}$ & $46 \mathrm{~m}$ & $14(11) \mathrm{m}$ & $25 \mathrm{~m}$ & 1.067 \\
3 & $600 \mathrm{~m}$ & $350 \mathrm{~m}$ & $80 \mathrm{~m}$ & $30(20) \mathrm{m}$ & - & 0.909 \\
\hline
\end{tabular}

TABLE 2. Distance $\left(D_{\text {source }}\right)$ between the generation site and the observation position, measured along the ship's track, distance $\left(D_{\text {track }}\right)$ from observation position to track, depth before depth change $\left(h_{A}\right)$, depth of shallow region $\left(h_{B}\right)$, upstream depth $\left(h_{C}\right)$ and depth change ratio $\Delta h / h=2\left(h_{A}-h_{B}\right) /\left(h_{A}+h_{B}\right)$ at observation positions $1-3$.

surface:

$$
\partial \eta / \partial t=\partial \phi / \partial y, \quad \partial \phi / \partial t+g \eta=-p / \rho, \quad y=0,
$$

where $\eta$ denotes the elevation, $\phi$ velocity potential, $p$ pressure distribution and $\rho$ density. The bottom variation is given by $y_{B}=-h+\beta(\mathbf{x})$ where $h$ is an average depth and $\beta$ the depth variation. We introduce

$$
\phi_{F}(\mathbf{x})=\phi(\mathbf{x}, y=0), \quad \phi_{B}(\mathbf{x})=\phi\left(\mathbf{x}, y=y_{B}\right), \quad V=\partial \phi /\left.\partial y\right|_{y=0},
$$

where $\phi_{F}$ denotes the potential evaluated at the free surface, $\phi_{B}$ potential along the bottom and $V$ the normal velocity at the free surface. The normal velocity at the bottom is zero. The functions $\phi_{F}, \phi_{B}$ and $V$ are connected through solution of the Laplace equation and is expressed in form of a set of integral equations. The solution method is an extension of Clamond and Grue $(2001, \S 6)$, Grue $(2002, \S 6)$, Fructus and Grue (2007, $\S 3)$ and Grue (2015). An evaluation point on the free surface $\left(y^{\prime}=0\right)$ gives

$$
\int_{F}\left(\frac{1}{r}+\frac{1}{r_{1}}\right) \frac{\partial \phi}{\partial n} d S=2 \pi \phi_{F}\left(\mathbf{x}^{\prime}\right)+\int_{F+B} \phi \frac{\partial}{\partial n}\left(\frac{1}{r}+\frac{1}{r_{1}}\right) d S
$$

where $1 / r=\left(R^{2}+\left(y^{\prime}-y\right)^{2}\right)^{-1 / 2}$ denotes a source function, $R=\left|\mathbf{x}^{\prime}-\mathbf{x}\right|$ horizontal distance, $1 / r_{1}=\left(R^{2}+\left(y^{\prime}+y+2 h\right)^{2}\right)^{-1 / 2}$ the image with respect to $y^{\prime}=-h, F$ the free surface, $B$ bottom of the fluid layer and $d S$ integration element. The normal $n$ points out of the fluid. A prime indicates evaluation variable where $\phi^{\prime}=\phi\left(\mathbf{x}^{\prime}, y^{\prime}\right)$ etc. For an evaluation point at the bottom $\left(y^{\prime}=-h+\beta^{\prime}\right)$ we obtain

$$
2 \pi \phi_{B}^{\prime}\left(\mathbf{x}^{\prime}\right)=\int_{F}\left(\frac{1}{r}+\frac{1}{r_{1 B}}\right) \frac{\partial \phi}{\partial y} d S-\int_{B} \phi_{B} \frac{\partial}{\partial n}\left(\frac{1}{r}+\frac{1}{r_{1 B}}\right) d S,
$$

where $1 / r_{1 B}=\left(R^{2}+\left(y^{\prime}+y\right)^{2}\right)^{-1 / 2}$. Solution of (3.3)-(3.4) is obtained expanding $1 / r$ and its images in powers of the bottom excursion $\beta$. Consider the integral over $F$ in $(3.4)$ where the function $1 / r+1 / r_{1 B}$ is expressed by

$$
\frac{1}{r}+\frac{1}{r_{1 B}}=2\left(1-\beta^{\prime} \frac{\partial}{\partial h}+\frac{1}{2 !}{\beta^{\prime}}^{2} \frac{\partial^{2}}{\partial h^{2}}-\frac{1}{3 !} \beta^{\prime 3} \frac{\partial^{3}}{\partial h^{3}}+\ldots\right) \frac{1}{R_{0}}, \quad R_{0}^{2}=R^{2}+h^{2} .
$$

Note that $1 / R_{0}=\mathcal{F}^{-1}\left[(2 \pi / k) e^{-\mathrm{i} \mathbf{k} \cdot \mathbf{x}-k h}\right]$ where $\mathcal{F}$ denotes Fourier transform, $\mathcal{F}^{-1}$ inverse transform, $\mathbf{k}=\left(k_{1}, k_{2}\right)$ wavenumber vector in Fourier space and $k=|\mathbf{k}|$. This gives for the integral over $F$ in $(3.4)$

$$
\frac{1}{2 \pi} \int_{F}\left(\frac{1}{r}+\frac{1}{r_{1 B}}\right) \frac{\partial \phi}{\partial y} d S=\mathcal{F}^{-1}\left[\frac{2 \hat{V} e_{1}}{k}\right]+\frac{1}{1 !} \beta^{\prime} \mathcal{F}^{-1}\left(2 \hat{V} e_{1}\right)+\frac{1}{2 !} \beta^{\prime 2} \mathcal{F}^{-1}\left(2 k \hat{V} e_{1}\right)
$$




$$
+\frac{1}{3 !} \beta^{\prime 3} \mathcal{F}^{-1}\left(2 k^{2} \hat{V} e_{1}\right)+. .
$$

where $e_{1}=e^{-k h}$. Similarly the integrals over $B$ in (3.4) are expressed by:

$$
\begin{aligned}
& -\int_{B} \phi_{B} \frac{\partial}{\partial n} \frac{1}{r} d S=\int_{B} \nabla_{1} \phi_{B} \cdot\left(\left[\left(\beta-\beta^{\prime}\right)-\frac{1}{3 !}\left(\beta-\beta^{\prime}\right)^{3} \nabla_{1}^{2}+\frac{1}{5 !}\left(\beta-\beta^{\prime}\right)^{5} \nabla_{1}^{4}+\ldots\right]\right) \nabla_{1} \frac{1}{R} d \mathbf{x}, \\
& -\int_{B} \phi_{B} \frac{\partial}{\partial n} \frac{1}{r_{1 B}} d S=-\int_{B} \phi_{B} \frac{\partial}{\partial(2 h)} \frac{1}{R_{1}} d \mathbf{x} \\
& +\int_{B} \nabla_{1} \phi_{B} \cdot\left(\frac{1}{1 !}\left(\beta+\beta^{\prime}\right)-\frac{1}{2 !}\left(\beta+\beta^{\prime}\right)^{2} \frac{\partial}{\partial(2 h)}+\frac{1}{3 !}\left(\beta+\beta^{\prime}\right)^{3} \frac{\partial^{2}}{\partial(2 h)^{2}}-\ldots\right) \nabla_{1} \frac{1}{R_{1}} d \mathbf{x},
\end{aligned}
$$

where in the latter $R_{1}^{2}=R^{2}+4 h^{2}$ and $\nabla_{1}^{\prime}=\left(\partial / \partial x_{1}^{\prime}, \partial / \partial x_{2}^{\prime}\right)$ denotes horizontal gradient. Using that $1 / R=\mathcal{F}^{-1}\left[(2 \pi / k) e^{-\mathbf{i} \mathbf{k} \cdot \mathbf{x}}\right]$ and $1 / R_{1}=\mathcal{F}^{-1}\left[(2 \pi / k) e^{-\mathbf{i} \mathbf{k} \cdot \mathbf{x}-2 k h}\right]$ the resulting set of equations for $\phi_{B}$ are obtained by (A 4)-(A 5) in Appendix A.3. By similar procedures the integral over $B$ in the last term in (3.3) becomes

$$
\frac{1}{2 \pi} \mathcal{F}\left(\int_{B} \frac{\partial}{\partial n}\left(\frac{1}{r}+\frac{1}{r_{1}}\right) \phi_{B} d S\right)=\frac{2 e^{-k h} \mathrm{ik}}{k} \cdot\left[\mathcal{F}\left(\beta \nabla_{1} \phi_{B}\right)+\frac{k^{2}}{3 !} \mathcal{F}\left(\beta^{3} \nabla_{1} \phi_{B}\right)+\ldots\right],
$$

while the surface integrals on the l.h.s. and r.h.s. of (3.3) become $2 \pi \hat{V}\left(1+e_{1}^{2}\right) / k$ and $-2 \pi \hat{\phi}_{F} e_{1}^{2}$, respectively, giving

$$
\hat{V}=k T_{1} \hat{\phi}_{F}+\left(\frac{1}{1 !} \hat{A}_{1}+\frac{k^{2}}{3 !} \hat{A}_{3}+\ldots\right) \frac{1}{C_{1}},
$$

where $T_{1}=\tanh k h, C_{1}=\cosh k h$ and $\hat{A}_{n}=\mathrm{ik} \cdot \mathcal{F}\left(\beta^{n} \nabla_{1} \phi_{B}\right)$. The resulting set of equations expresses $\left(V, \phi_{B}\right)$ in terms of the surface potential $\phi_{F}$ and expansions of the bottom variation ordered according to $\beta^{n}(n=0,1,2, \ldots)$ where the smallness parameter is $\max (\beta / h)=O\left(\frac{1}{2} \Delta h / h\right)$. The set of variables of the leading two orders become

$$
\left(k \hat{\phi}_{B}^{(0)}, \hat{V}^{(0)}\right)=\left(k \hat{\phi}_{F} / C_{1}, k T_{1} \hat{\phi}_{F}\right), \quad\left(k \hat{\phi}_{B}^{(1)}, \hat{V}^{(1)}\right)=\left(-T_{1} \hat{A}_{1}, \hat{A}_{1} / C_{1}\right) .
$$

Regarding the vertical velocity we obtain that $\hat{V}^{(2)}=0$ and $\hat{V}^{(3)}=k^{2} \hat{A}_{3} /\left(6 C_{1}\right)$ where $T_{1}, C_{1}, \hat{A}_{n}$ are defined below (3.10). The representations $\phi_{B}^{(0)}+\phi_{B}^{(1)}+\phi_{B}^{(2)}+\phi_{B}^{(3)}$ and $\hat{V}^{(0)}+\hat{V}^{(1)}+\hat{V}^{(3)}$ are obtained iteratively from (3.11) and (A 4)-(A 5) in Appendix A.3. We define the truncation level of the approximations of $\left(\phi_{B}, V\right)$ by

$$
\begin{aligned}
& s^{(1)}=\left(\phi_{B}^{(0)}+\phi_{B}^{(1)}, V^{(0)}+V^{(1)}\right), \\
& s^{(2)}=\left(\phi_{B}^{(0)}+\phi_{B}^{(1)}+\phi_{B}^{(2)}, V^{(0)}+V^{(1)}\right), \\
& s^{(3)}=\left(\phi_{B}^{(0)}+\phi_{B}^{(1)}+\phi_{B}^{(2)}+\phi_{B}^{(3)}, V^{(0)}+V^{(1)}+V^{(3)}\right),
\end{aligned}
$$

where convergence of the computations is illustrated using the approximations $s^{(2)}$ and $s^{(3)}$, see $\S 5$ below. The approximation $s^{(2)}$ is computationally less expensive than $s^{(3)}$.

The resulting equation system for the free surface variables $\eta$ and $\phi_{F}$ is expressed by Fourier transform

$$
\frac{\partial \hat{\eta}}{\partial t}=k T_{1} \hat{\phi}_{F}+\hat{h}_{1}, \quad \frac{\partial \hat{\phi}_{F}}{\partial t}+g \hat{\eta}=\hat{h}_{2}
$$

where $\hat{h}_{1}=\hat{A}_{1} / C_{1}+k^{2} \hat{A}_{3} /\left(6 C_{1}\right)+\ldots$ is obtained from $(3.10)$ and $\hat{h}_{2}=-\hat{p} / \rho$ is given by the moving pressure distribution. Time integration of (3.15) is expressed on matrix 
form: $\hat{Y}_{t}+A \hat{Y}=\hat{H}$ where $\hat{Y}=\left[\hat{\eta},(\omega / g) \hat{\phi}_{F}\right]^{T}, \hat{H}=\left[\hat{h}_{1}, \omega \hat{h}_{2} / g\right]^{T}, \omega^{2}=g k \tanh k h$ and []$^{T}$ denotes transpose. The matrix $A$ and its variant $e^{A s}$ are obtained by

$$
A=\left[\begin{array}{cc}
0 & -\omega \\
\omega & 0
\end{array}\right], \quad e^{A s}=\left[\begin{array}{cc}
\cos \omega s & -\sin \omega s \\
\sin \omega s & \cos \omega s
\end{array}\right] .
$$

Time-integration of $\left(e^{A t} \hat{Y}\right)_{t}=e^{A t} \hat{H}$ gives $\hat{Y}=\int_{t_{0}}^{t} e^{A(s-t)} \hat{H} d s$ where no elevation, motion or pressure impulse for $t_{0}<0$ are assumed. The elevation is obtained by

$$
\hat{\eta}=\int_{t_{0}}^{t} \cos \omega(s-t) \hat{h}_{1}(s) d s+\int_{t_{0}}^{t} \sin \omega(s-t) \frac{\omega \hat{p}}{\rho g} d s
$$

where the first term accounts for the effect of the bottom variation and the second term the effect of the pressure distribution moving along a fluid layer of constant water depth $h$. Eq. (3.17) is used in the asymptotic analysis in $\S 4$.

\section{Asymptotic analysis for long waves}

The very long upstream ship waves motivate for an asymptotic analysis valid for $k h<<$ 1. The long wave analysis is complemented by fully dispersive calculations in $\S 5$ below. Wave generation due to a depth change at $x_{1}=0$ is considered, where the water depth is assumed to be $h$ for $x_{1}<0$ and $h-\Delta h$ for $x_{1}>0$, with $\Delta h / h<<1$. This means that $\beta=0$ for $x_{1}<0$ and $\beta=\Delta h$ for $x_{1}>0$. For small $k h$ we have from (3.11) $\hat{\phi}_{B} \simeq \hat{\phi}_{B}^{(0)} \simeq \hat{\phi}_{F}$ where $\cosh k h \simeq 1$ is used. Evaluating the Fourier transform we obtain for $\hat{h}_{1}$ :

$$
\hat{h}_{1} \simeq \mathrm{i} k_{1} \Delta h \int_{0}^{\infty} \int_{-\infty}^{\infty} \frac{\partial \phi_{F}}{\partial x_{1}} e^{-\mathrm{i} \mathbf{k} \cdot \mathbf{x}} d \mathbf{x}+O\left(k^{2}\right) \simeq-\mathrm{i} k_{1} \Delta h \int_{-\infty}^{\infty} \phi_{F}\left(x_{1}=0\right) d x_{2}+O\left(k^{2}() \cdot 1\right)
$$

where partial integration in the $x_{1}$-direction has been used. This obtains the leading contribution to the long wave formation by the wave potential integrated laterally along the step. The result (4.1) may alternatively be obtained directly from the integral equation formulation, see (A 3) in A.2. Time derivative of (4.1) gives:

$$
\frac{\partial \hat{h}_{1}}{\partial t} \simeq-\mathrm{i} k_{1} \Delta h \int_{-\infty}^{\infty} \frac{\partial \phi_{F}\left(x_{1}=0\right)}{\partial t} d x_{2} \simeq \frac{\mathrm{i} k_{1} \Delta h}{\rho} \int_{-\infty}^{\infty} p\left(x_{1}=0\right) d x_{2},
$$

where the dynamic boundary condition at the free surface is used. By partial integration in time the first term in (3.17), which we denote by $\hat{\eta}_{0}$, becomes,

$$
\hat{\eta}_{0}=-\int_{t_{0}}^{t} \frac{\sin \omega(s-t)}{\omega} \frac{\partial \hat{h}_{1}}{\partial s} d s \simeq-\frac{\mathrm{i} k_{1} \Delta h}{\rho} \int_{t_{0}}^{t} \frac{\sin \omega(s-t)}{\omega} \int_{-\infty}^{\infty} p\left(x_{1}=0\right) d x_{2} d s,
$$

where (4.2) has been used. Eq. (4.3) expresses $\hat{\eta}_{0}$ in terms of a pressure impulse acting at the depth change caused by the moving pressure distribution. By assuming that the pressure, moving with constant speed $U$ along the $x_{1}$-direction, is modelled by the Dirac delta function in the two horizontal directions, i.e., $p\left(x_{1}-U t, x_{2}, t\right)=\rho g V_{0} \delta\left(x_{1}-U t\right) \delta\left(x_{2}\right)$, we obtain

$$
\hat{\eta}_{0}=\frac{\mathrm{i} k_{1} \Delta h V_{0}}{U \omega / g} \sin \omega t,
$$

where $V_{0}$ is the volume of the pressure distribution. For a distributed pressure, symmetrical in $x_{1}$ and $x_{2}$, the result (4.4) is multiplied by a function $f(\kappa)=$ 
$\int_{-l_{0} / 2}^{l_{0} / 2} d s^{*} \cos \left(\omega s^{*} / U\right) \times \times \int_{-w\left(x_{1}\right) / 2}^{-w\left(x_{1}\right) / 2} d x_{2} \tilde{d}\left(-s^{*}, x_{2}\right)$ where $\kappa=\omega l_{0} /(2 U), \quad l_{0}$ is the length, $w\left(x_{1}\right)$ local width and $\tilde{d}$ the shape function of the pressure. Assuming, e.g., $\left.\tilde{d}=d_{0}\left(1-\left(2 x_{1} / l_{0}\right)^{2}-\left(2 x_{2} / w_{0}\right)^{2}\right)\right)$ we obtain $f(\kappa)=f_{0}(\kappa)=8 J_{2}(\kappa) / \kappa^{2}$ where $J_{2}$ denotes the Bessel function of the first kind of order two, and is used for illustrative purposes in figure 3 .

We first investigate the motion along a narrow channel where the far field motion can be considered as two-dimensional. (The two-dimensional analysis involves Fourier transform in the $x_{1}$-direction only, however, the result (4.4) is unchanged. The volume $V_{0}$ is two-dimensional.) Inverse transform of (4.4) gives

$$
\eta_{0}\left(x_{1}, t\right)=-\frac{\Delta h V_{0}}{2 \pi U / g} \int_{0}^{\infty} \frac{k_{1}}{\omega} \cos \left(k_{1} x_{1}-\omega t\right) d k_{1}+\frac{\Delta h V_{0}}{2 \pi U / g} \int_{0}^{\infty} \frac{k_{1}}{\omega} \cos \left(k_{1} x_{1}+\omega t\right) d k(1.5)
$$

The upstream waves are obtained by the method of stationary phase for $t \rightarrow \infty$, giving,

$$
\eta_{0}\left(x_{1}, t\right)=-\frac{k_{1,0} \Delta h V_{0}}{\left(U \omega_{0} / g\right)\left(2 \pi\left|\omega^{\prime \prime}\right| t\right)^{1 / 2}} \cos \left(k_{1,0} x_{1}-\omega_{0} t+\frac{\pi}{4}\right)+O\left(t^{-1}\right) .
$$

Here, $k_{1,0}$ is determined by $\partial \omega / \partial k_{1}=x_{1} / t<\sqrt{g h}=c_{0}, \omega_{0}=\omega\left(k_{1,0}\right)$ and a double prime means double derivative. For $x_{1} / t \rightarrow \sqrt{g h}$ both $k_{1,0} \rightarrow 0$ and $\omega_{0}^{\prime \prime} \rightarrow 0$, and the asympototic expression (4.6) diverges. For $x_{1} / t>\sim \sqrt{g h}$ the first term in eq. (4.5) is then expressed in terms of the Airy integral where the phase function for small $k_{1}$ is expanded by $k_{1} x_{1}-\omega t \simeq k_{1}\left(x-c_{0} t\right)-c_{0} h^{2} k_{1}^{3} t / 6+\ldots$ Introducing the variables $Z=\left[2\left(x_{1}-c_{0} t\right)^{3} / c_{0} h^{2} t\right]^{1 / 3}$ and $k_{1}\left(x_{1}-c_{0} t\right)=Z \alpha$, following Mei (1989), we obtain

$$
\eta_{0}\left(x_{1}, t\right) \sim-\frac{\Delta h V_{0}}{\left(U c_{0} / g\right)\left(4 c_{0} h^{2} t\right)^{1 / 3}} \operatorname{Ai}(Z),
$$

where $\operatorname{Ai}(Z)$ denotes the Airy function.

Inverse transform of (4.4) in the three-dimensional case gives:

$$
\eta_{0}\left(x_{1}, x_{2}, t\right)=\frac{\Delta h V_{0}}{4 \pi^{2} U / g} \frac{\partial}{\partial x_{1}} \int_{0}^{\infty} \int_{0}^{2 \pi} e^{\mathrm{i} k R \cos (\alpha-\gamma)} \frac{\sin \omega t}{\omega} k d \alpha d k,
$$

where $\left(x_{1}, x_{2}\right)=R(\cos \gamma, \sin \gamma)$ and $\left(k_{1}, k_{2}\right)=k(\cos \alpha, \sin \alpha)$ are introduced. The inner integral in (4.8) is $J_{0}(k R)$, the Bessel function of the first kind of order zero, giving

$$
\eta_{0}\left(x_{1}, x_{2}, t\right)=\frac{\Delta h V_{0}}{2 \pi U / g} \frac{\partial}{\partial x_{1}} \int_{0}^{\infty} \frac{\sin \omega t}{\omega} J_{0}(k R) k d k .
$$

Investigating the contribution for large $k R$ where asymptotically $J_{0}(k R) \sim(2 /(\pi k R))^{1 / 2} \times$ $\times \cos (k R-\pi / 4)$, we obtain

$\eta_{0}(R, t) \sim \frac{\Delta h V_{0}}{4 \pi U / g} \frac{\partial}{\partial x_{1}} \int_{0}^{\infty} \frac{k}{\omega}\left(\frac{2}{\pi k R}\right)^{1 / 2}\left(-\sin \left(k R-\omega t-\frac{\pi}{4}\right)+\sin \left(k R+\omega t-\frac{\pi}{4}\right)\right) d k$.

The asymptotic elevation, obtained by the method of stationary phase for large $R$ and $t$, becomes

$$
\eta_{0}(R, t) \sim-\frac{\Delta h V_{0} k_{0}}{2 \pi U \omega_{0} / g} \frac{\partial}{\partial x_{1}} \frac{\sin \left(k_{0} R-\omega_{0} t\right)}{\left(k_{0} R\left|\omega_{0}^{\prime \prime}\right| t\right)^{1 / 2}},
$$

where $k_{0}$ is determined by $R / t=\partial \omega / \partial k<\sqrt{g h}, \omega_{0}=\omega\left(k_{0}\right)$ and a double prime means double derivative.

Eq. (4.11) is not valid ahead of the wave front. Clarisse, Newman and Ursell (1995, 
eq. (6.15)) have developed a second-order asymptotic expression for the integral $\int_{0}^{\infty}(1 / \omega) \sin \omega t J_{0}(k R) k d k$ in $(4.9)$ valid for $R / t \sim \sqrt{g h}$, giving for the present wave field $\eta_{0}\left(x_{1}, x_{2}, t\right) \sim \frac{\Delta h V_{0} / h^{2}}{2 \pi U / \sqrt{g h}} \frac{\partial}{\partial x_{1}} \frac{2^{1 / 3} \pi}{\left(R^{*} / t^{*}\right)^{1 / 2} t^{* 2 / 3}}\left[E_{0} \mathrm{Ai}^{2}-t^{*-2 / 3} C_{0} \mathrm{Ai}^{\prime 2}+2 t^{*-4 / 3} A_{1} \mathrm{AiAi}^{\prime}\right]$.

The argument of the Airy function $\mathrm{Ai}$ and its derivative $\mathrm{Ai}^{\prime}$ is specified below Clarisse et al. (1995, eq. (6.15)) and $R^{*}=R / h$ and $t^{*}=t \sqrt{g / h}$. The functions $E_{0}$ and $C_{0}$ are obtained from saddle points 1 and 2 of the phase function of their asympotic integral, see Clarisse et al. (1995, eq. (5.4)), and are evaluated here.

The asymptotic analysis provides in mathematical terms a qualitative explanation of the upstream wave generation which by (4.3) is expressed in terms of a pressure impulse acting at the bottom step. The leading waves propagate with the shallow water speed $c_{0}=\sqrt{g h}$ where the wave amplitude is proportional to the depth change $\Delta h$ times the volume $V_{0}$ of the ship. In the three-dimensional case the wave crests are circular. The amplitude is multiplied by the cosine of the wave angle defined from direction of motion of the pressure and appears because of the derivative $\partial / \partial x_{1}$ in (4.11). For a positive $\Delta h$, where the depth is reduced from $h$ to $h-\Delta h$, the leading upstream wave is a depression, while the downstream waves have a positive leading elevation, both in twoand three-dimensions. A leading upstream wave of depression at a depth change from deep to shallow water is in accordance with the video recording at the observation position 3 at Askholmene (https://www.youtube.com/watch?v=42Ctdk9kpyg). The conclusion is opposite for a negative step with $\Delta h<0$. Upstream wave trains obtained by the asymptotics in two and three dimensions are visualized in figure $3(\Delta h>0)$.

While (4.4) and the subsequent derivations are based on a localized effect of the pressure impulse in the form of a delta function, a distributed pressure modifies the far field waves. Results multiplying the two-dimensional wave amplitude in (4.6) by the function $f_{0}\left(\kappa_{0}\right)=8 J_{2}\left(\kappa_{0}\right) / \kappa_{0}^{2}$, with $\kappa_{0}=\omega_{0} l_{0} /(2 U)$, as obtained below (4.4), with $l_{0} / h=3.82$, a length ratio fitting with the conditions discussed in $\S 5$, are included in figure $3 \mathrm{a}, \mathrm{b}$, for $F r=0.4$ and 0.5 . Apart from the leading trough, the amplitude of the modified far field waves exhibit a weak increase with the Froude number.

\section{Fully dispersive calculations}

Calculations of fully dispersive wave fields complement the asymptotic analysis in $\S 4$ and are connected to the observations in $\S 2$. The equation system $\left(e^{A t} \hat{Y}\right)_{t}=e^{A t} \hat{H}$ defined in the text above and below (3.16) is integrated forward in time using a fourth order Runge-Kutta scheme (RK4) (with time step $\Delta t \sqrt{g / h}=0.025$ ) where the moving pressure distribution $p\left(x_{1}-x_{1,0}(t), x_{2}\right)$ is specified below. The coordinate $x_{1,0}(t)$ gives the forward motion where $U=\dot{x}_{1,0}(t)$. The numerical discretization is obtained over horizontal rectangles of length and width of $L_{1}$ and $L_{2}$, respectively, with $N_{1}$ by $N_{2}$ computational points in each respective direction and a resolution of $\left(\Delta x_{1}, \Delta x_{2}\right)=\left(L_{1} / N_{1}, L_{2} / N_{2}\right)$. Use of Fourier transform implies that the computational domain is periodic in both directions, where a length $L_{1}$ much greater than width $L_{2}$ in practice models a long channel with straight walls. The finite length $L_{1}$ puts limits on the length of the time simulation where the calculations are stopped before the motion interacts with itself. The wavenumber vectors in spectral space read $\left(0, \Delta k_{1}, 2 \Delta k_{1}, \ldots,\left(N_{1} / 2-1\right) \Delta k_{1}\right.$, $\left.-\left(N_{1} / 2\right) \Delta k_{1}, \ldots,-\Delta k_{1}\right)$ and $\left(0, \Delta k_{2}, 2 \Delta k_{2}, \ldots,\left(N_{2} / 2-1\right) \Delta k_{2}\right)$ where symmetry in the $k_{2}$-direction is assumed. The number of operations of the method is governed by the 
FFT-operations which is $\mathcal{O}\left(N_{0} \log N_{0}\right)$, where $N_{0}=N_{1} \cdot N_{2}$, the number of calculation nodes, is up to $N_{0}=720 \times 720 \simeq 0.5 \cdot 10^{6}$ using a Lenovo T430 thinkpad.

\subsection{Bottom step in a moderately narrow channel}

The bottom profile along the $x_{1}$-direction is assumed to be given by the function

$$
\beta=-\frac{1}{2} \Delta h+\frac{1}{2} \Delta h\left[\tanh \left(\alpha_{0}\left(x_{1}-x_{1, a}\right)\right)-\tanh \left(\alpha_{0}\left(x_{1}-x_{1, b}\right)\right)\right],
$$

where $x_{1, a}$ denotes the center position of the depth change, $x_{1, b}$ the similar position where the water gets deep again, and $\alpha_{0}$ is either 0.7 or 2.0, see figure 4 . A uniform depth in the lateral direction is assumed. The applied surface pressure, given here by $p\left(x_{1}, x_{2}\right)=p_{0} \exp \left(-\left(2 x_{1} / l_{0}\right)^{6}-\left(2 x_{2} / w_{0}\right)^{6}\right)$, where $l_{0}$ and $w_{0}$ denote length and width, respectively, is connected to the ship volume $V_{0}$ by $\iint p d x_{1} d x_{2}=\rho g V_{0}$. The velocity $\dot{x}_{1,0}(t)$ of the moving pressure has a gentle ramp up phase generating minimal upstream waves $\left(\dot{x}_{1,0}(t)=U \sin \left(t / T_{0}\right), T_{0} \sqrt{g / h}=150, x_{1,0}(t=0)=6 h\right)$.

With reference to the conditions at the observation position 3 (table 2) a reference depth of $h=55 \mathrm{~m}$ and depth change ratio of $\Delta h / h=0.909$ are used. The nondimensional volume and length of the largest among the ships in table 4 in Appendix A.1 become $V_{0}^{\prime}=$ $V_{0} / h^{3} \simeq 0.216$ and $l_{0} / h \simeq 3.82$, respectively. Two different computational rectangles are used, the first, for $\Delta h>0$ : $\left(L_{1}, L_{2}\right)=(200 h, 13 h),\left(N_{1}, N_{2}\right)=(1400,200), x_{1, a}=60$, $x_{1, b}=180$, and, the second, for $\Delta h<0:\left(L_{1}, L_{2}\right)=(320 h, 13 h),\left(N_{1}, N_{2}\right)=(1800,200)$, $x_{1, a}=80, x_{1, b}=300$. Note that the width of the numerical channel of $L_{2}=13 h$ corresponds to twice the distance between the observation position 3 and the ship's track.

A typical ship speed of $\dot{x}_{1,0}=U=10 \mathrm{~ms}^{-1}$ gives $F r=U / \sqrt{g h} \simeq 0.43$ where simulations are carried out for $F r \sim 0.26-0.51$. The elevation ahead of the ship, comparing the two approximations $s^{(2)}$ and $s^{(3)}$ given in (3.13-3.14), is obtained for $\Delta h>0, F r=0.43$ and $t^{*}=281$ in figure 5a. Results for the case with $\Delta h<0$ are shown for $F r=0.51, t^{*}=260$ in figure $5 \mathrm{~b}$. Both sets of results are obtained with a bottom slope of $\alpha_{0}=0.7$, where the very small difference between the two approximations illustrates the convergence of the method. Note that the approximations $s^{(2)}$ and $s^{(3)}$ model short wave effects differently, e.g., the bow wave of the pressure distribution, see figure $5 \mathrm{c}, \mathrm{d}$. The entire computational domain is shown in figure 5e for $\Delta h<0, t^{*}=293$. The upstream wave due to a slope factor of $\alpha_{0}=2.0$, obtained for $F r=0.43, t^{*}=281$, is somewhat ahead of the wave produced with a slope factor of $\alpha_{0}=0.7$. The wave is also somewhat higher (figure 5a). Otherwise differences are minor compared to the bottom profile with $\alpha_{0}=0.7$.

The generation during the interaction with the depth change at $x_{1, a}=60 \mathrm{~h}$ is visualized for $F r=0.43$ in figure 6 at times $t^{*}=211,223,246$ and in figure $5 \mathrm{c}, \mathrm{d}$ for $t^{*}=281$. The main wave quickly extends laterally across the channel. It is basically characterized as a one-dimensional hump in straight forward motion. The propagation

speed is $0.994 \sqrt{g\left(h-\frac{1}{2} \Delta h\right)}$, between $t^{*}=246$ and 281. Succeeding shorter waves along the channel walls are observed (figure $5 \mathrm{c}, \mathrm{d}$ ). When the depth changes from shallow to deep water, the similar wave is a depression. The speed is $0.96 \sqrt{g(h+\Delta h)}$, right ahead of the ship, between $t^{*}=281$ and 293 , where the negative elevation $(-\eta)$ is plotted in figure 7 for times $t^{*}$ between 251 and 293 .

The main wave is always succeeded by a train of shorter waves of small amplitude. A leading upstream elevation of small distinct amplitude preceeds the main depression wave in the case when the depth changes from shallow to deep water $(\Delta h<0)$ (figure $8 \mathrm{c}$ ). This is in accordance with the asymptotics derived in $\S 4$. However, for positive $\Delta h>0$, 
a corresponding leading depression is really tiny, where the computed surface for time $t^{*}=281$ in figure $8 \mathrm{a}$ is only slightly below the level at the earlier times $t^{*}=223,246$.

The main upstream elevation or depression clearly depend on the forward speed (figure 8b,c). The maximum elevation, $\eta_{\max }$, for $\Delta h>0$, and the minimum elevation, $\eta_{\min }$, for $\Delta h<0$, both grow according to $F r^{n}$, with $n \simeq 3.2$ for $\eta_{\max }$ and $n \simeq 4$ for $\eta_{\min }$ (figure $9 \mathrm{a}$ ). We define the wave width at half crest height, at $y=\frac{1}{2} \eta_{\max }$, by $\lambda_{1 / 2}^{c r}$, when the bottom changes from deep to shallow water, and similarly, the wave

width at half trough height, at $y=\frac{1}{2} \eta_{\min }$, by $\lambda_{1 / 2}^{t r}$, when the bottom changes from shallow to deep water. The wave widths in the range of $6.5 h-12 h$ decay with increasing Fr (figure 9b). The symmetry between the dominant upstream wave for positive and negative $\Delta h$ is illustrated by forming the products $\eta_{\max } \lambda_{1 / 2}^{c r}$ and $-\eta_{\min } \lambda_{1 / 2}^{t r}$. These become approximately equal and exhibit a common algebraic growth in the Froude number, in these calculations (figure 9c).

The calculations are compared to the observations at position 3. A period of approximately 32 seconds of the leading two main waves can be estimated from the videorecording of the flow in the bay at Askholmene. Multiplying by the shallow water speed, this gives a wavelength of about $550 \mathrm{~m}$ corresponding to $\sim 10 \mathrm{~h}$. The calculated wavelength for $\mathrm{Fr} \sim 0.43$ at $t^{*}=281$ in figure 8 a corresponds fairly well to the observation. Calculated maximal nondimensional wave elevations of $\sim 0.0013 \mathrm{~h}(\mathrm{Fr}=$ $0.43)$ and $\sim 0.0021 h(F r=0.49)$ in figure 8a correspond to amplitudes of $7 \mathrm{~cm}$ and 12 $\mathrm{cm}$ in the fjord, respectively, of the main wave ahead of the larger among the ships, of displaced volume of $36000 \mathrm{~m}^{3}$. The small elevations generate run-ups and run-downs of the order of $1 \mathrm{~m}$ along the shores, as evidenced from the movie. The calculations show one dominant elevation and succeeding minor shorter waves along the channel wall, comparing rather well to the short wave train which is typical for the observations at position 3.

The elevation ratio $\eta^{\prime}=\eta L_{2} /\left(\Delta h V_{0} / h^{2}\right)$ and wave width $\lambda^{1 / 2}$ are compared to the asymptotics in $\S 4$. The dispersive calculation in figure $8 \mathrm{~b}$ for $F r=0.51, t^{*}=280$ corresponds to a time $t^{*}-t_{a}^{*} \simeq 88.5$ upstream of the bottom transition at $x_{1, a}$. Similarly, the calculation in figure $8 \mathrm{c}$ for $F r=0.51, t^{*}=260$ corresponds to a time $t^{*}-t_{a}^{*} \simeq 29.3$ upstream of $x_{1, a}$. Table 3 compares the wave height $\eta_{\text {max }}^{\prime}-\eta_{\text {min }}^{\prime}$, measured as the height from the first elevation to the second trough, for $\Delta h>0$, and the height from the first trough to the second crest, for $\Delta h<0$. The table also compares $\lambda^{1 / 2}$ of the leading main wave in figures $8 \mathrm{~b}, \mathrm{c}$ to those obtained by the asymptotics in the two-dimensional case, in figure $3 \mathrm{a}, \mathrm{b}$, where the latter is measured at $y=0$. Apart from some differences in the wavelength for $\Delta h<0$, the comparison is rather good. Both the crest, trough and wave height increase with $F r$, where the growth is weak for $\Delta h / h<<1$ (the asymptotics), and very strong for $\Delta h / h \simeq 1$. The leading trough (crest) for $\Delta h>0(\Delta h<0)$, pronounced for $\Delta h / h<<1$, but either weak or almost absent for $\Delta h / h \simeq 1$, is another difference.

\section{Upstream waves in a wide channel}

A channel of width of $2.4 \mathrm{~km}$ corresponds to twice the distance from the observation position 1 to the ship's track. The depth changes from moderately deep to shallow to moderately deep water, with $h=\frac{1}{2}\left(h_{A}+h_{B}\right)=30 \mathrm{~m}$ and $\Delta h / h=1.067$, where the depth right north of the shallow region is $h+\frac{1}{2} \Delta h$, while still further north average depths of the fjord are given in table 1 . The shallow region extends about $23 h$ along the track. The positions $x_{1, a}=60 h$ and $x_{1, b}=83 h$ define its extension in the numerical calculations. The computational domain is $\left(L_{1}, L_{2}\right)=(300 h, 80 h)$ with $\left(N_{1}, N_{2}\right)=$ 
Asymptotic Dispersive

\begin{tabular}{|c|c|c|c|c|}
\hline$t^{*}-t_{a}^{*}$ & 30 & 90 & 29.3 & 88.5 \\
$\Delta h / h$ & $<<1$ & $<<1$ & 0.909 & 0.909 \\
$\eta_{\max }^{\prime}-\eta_{\min }^{\prime}$ & 0.19 & 0.18 & 0.17 & 0.17 \\
$\lambda^{1 / 2} / h$ & 3.8 & 5.9 & 8.2 & 6.8 \\
\hline
\end{tabular}

TABLE 3. Wave height $\eta_{\text {max }}^{\prime}-\eta_{\text {min }}^{\prime}$, where $\eta^{\prime}=\eta L_{2} /\left(\Delta h V_{0} / h^{2}\right)$, and wave length at half height $\lambda^{1 / 2}$, of first main wave, obtained by asymptotic model (in $\left.2 \mathrm{D}\right)(\Delta h / h<<1, F r=0.5)$ and dispersive calculation $(\Delta h / h=0.909, F r=0.51)$. Time $t^{*}-t_{a}^{*}$ after passage of the depth change at $x_{1, a}$.

$(1200,432)$ computational points. A ship speed of $10 \mathrm{~ms}^{-1}$ corresponds to $\mathrm{Fr}=0.58$ where the range $F r \sim 0.3-0.7$ is explored. Note that $U / \sqrt{g\left(h-\frac{1}{2} \Delta h\right)}$ becomes critical in the shallow region for the largest speed $(F r=0.7)$. However, nonlinear effects, known to propagate laterally in the wide channel (Pedersen, 1988), are assumed to be small over the relatively short extension of the shallow region (of length $23 \mathrm{~h}$ ).

The ship volume $V_{0}=36000 \mathrm{~m}^{3}$ is made dimensionless by the reference depth $h=30$ $\mathrm{m}$, giving $V_{0} / h^{3}=1.33$. This is used in the calculations shown in figures $10-11$. Note that this nondimensional ship volume is about 6 times larger than the nondimensional ship volume at Askholmene, where the reference depth is $h=55 \mathrm{~m}$. The upstream waves calculated using the approximation $s^{(2)}$ in (3.13) exhibit a curved pattern where the dominant feature is a leading, large wave trough, preceeded and succeeded by less dominant crests. A train of shorter waves of smaller amplitude follows behind the leading wave motion. The leading waves ahead of the ship in the wide channel are similar to the upstream waves generated at the depth change from shallow to deep water. More precisely, the calculated elevation in the narrow channel is in the range $-0.13<\eta L_{2} /\left(\Delta h V_{0} / h^{2}\right)<0.04$, see figure $8 \mathrm{c}$. The latter corresponds to a wave height ahead of the ship of $H / h \simeq 0.003$ in the wide channel. A simple doubling gives $2 H / h \simeq 0.6 \cdot 10^{-2}$, fitting fairly well with the results in figure $11 \mathrm{~b}$, for the ship passing over the two depth changes in the wide channel, for the similar $\mathrm{Fr}=0.51$.

Wave reflections at the side walls, where the propagation speed is enhanced (results not shown), produce a complex wave pattern in the channel. The leading curved crests eventually straighten up. Note that the wave interaction with the channel walls is not related to the Mach reflection, since the waves in consideration are linear. Snapshots of the wave elevation at the side wall illustrate further the dominant trough and preceeding and succeeding crests (figure 11a). The dominant wave trough corresponds to the strong outflow observed in the harbor at observation position 1 , see figure 1 . The observation position 1 is located $50 h$ upstream of the generation site $\left(x_{1, b}\right)$ where the maximum wave height $H$ grows according to $F r^{n}$ where $n \simeq 3.7$ at the channel wall and $n \simeq 3.5$ ahead of the ship. The heigth is approximately $45-55 \%$ higher at the walls than ahead of the ship, at the similar $x_{1}$-position (figure $11 b$ ). We obtain $H / h \simeq 0.025$ at the channel wall, for $F r=0.7$, giving $H \simeq 0.75 \mathrm{~m}$ (with $h=30 \mathrm{~m}$ ) and is in the range of the observations in the harbor.

The calculated leading trough to trough period at $x_{1}-x_{1, b}=50 h$ becomes $T=$ $21.8 \sqrt{h / g} \simeq 38$ seconds for $F r=0.58$, and $T=24.2 \sqrt{h / g} \simeq 41$ seconds for the smaller $F r=0.52$. A calculated third crest to crest period becomes 15 seconds for $F r=0.58$. 
The periods of the idealized calculations are rather close to the observed periods at position 1 , see table 2 . Similar calculations at the channel wall at an upstream position of $x_{1}-x_{1, b}=83 h$ for the still smaller $F r=0.46$ gives a wave period of $T=26.2 \sqrt{h / g} \simeq 45$ seconds. This upstream position corresponds to observation position 2 in table 2 , where the numerical period is somewhat smaller compared to the observed period of 57 seconds, for the similar ship speed, where, however, the fjord is much shallower $(\sim 25 \mathrm{~m})$ than in the calculation. In summary, despite the rather ideal conditions of the numerical channel, with a constant water depth of $46 \mathrm{~m}$ outside the shallow region, the calculated wave height and periods agree fairly well to the observations at positions 1 and 2 in the Oslofjord. Note that effects due to local bathymetry, bottom slope or shape of the shoreline, not accounted for in the computations, contribute to the actual run-ups, run-downs as well as the wave period.

The results in figure $11 \mathrm{~b}$ are used to calculate the wave height produced by the two different ships at position 1, cruising with speeds of $8.6 \mathrm{~ms}^{-1}$ and $10.3 \mathrm{~ms}^{-1}$, respectively, see table 1 . The corresponding wave heights become $H / h \simeq 0.70 \cdot 10^{-2}$ and $1.36 \cdot 10^{-2}$. Multiplying the former by the volume of Pearl Seaways, which is 69 per cent of Magic/Fantasy (table 4), we obtain that the faster and larger ship makes a wave height of approximately three times higher compared to the slower and smaller ship. The wave height ratio becomes four times, for a sailing speed of $11.5 \mathrm{~ms}^{-1}$ of Fantasy/Magic which has been observed by the people living in Flaskebekk.

\section{Conclusions}

Ship generated very long upstream waves of appreciable amplitude is a new phenomenon and are recently observed in the Oslofjord in Norway. The waves are generated locally in time and space, at positions where the water depth along the ship's track changes by an appreciable amount $\Delta h$ that is comparable to a relatively shallow average depth $h$ at the position, which means that $\Delta h / h \simeq 1$. The change $\Delta h$ may be positive or negative. The waves, propagating with a speed close to the shallow water speed, ahead of the ships, observed as slow run-ups and run-downs in harbors and bays along the shore with periods in the range 30-60 seconds, bear many similarites with tsunamis, although at a smaller length scale. We have found it tempting to denote the waves as a minitsunami. The waves, $0.5-1 \mathrm{~km}$ long, are generated by conventional ships moving with a depth Froude number in the range $F r=U / \sqrt{g h} \sim 0.4-0.7$ and a Froude number based on the ship length $l$ of $F r_{l}=U / \sqrt{g l} \sim 0.17-0.24$.

The linear generation mechanism is mathematically explored by an asymptotic analysis valid for small $\Delta h / h$ and long waves. This shows that the pressure due to the moving ship acts as an impulsive force at the depth change and provides a qualitative explanation of the phenomenon, see (4.3). The analysis gives a leading upstream wave of depression when the water depth reduces, in accordance with observations. Conversely, an upstream leading wave of elevation is formed when the depth increases. A train of shorter and slower waves is subsequent.

A fully dispersive linear analysis valid for $\Delta h / h \simeq 1$ complements the asymptotics where the depth variation is similar to the conditions in the observations. Computations in a moderately narrow channel show that one dominant upstream wave of elevation is generated when the depth undergoes a reduction. A leading depression, which, however, is very tiny in the numerical computations, may be observed ahead of the dominant wave. A train of shorter waves of small amplitude travels behind the main elevation. In the case when the depth increases, a dominant upstream wave of depression is generated. This is preceeded by a long wave of elevation, of small, however, distinct amplitude. A 
train of short waves of small amplitude is succeeding. The wave generation shows minor sensitivity to the bottom slope at the depth change. The symmetry for positive and negative $\Delta h$ is pointed out.

Computations for a wide channel show curved crests. Reflections at the channel walls enhance the elevation as well as the propagation speed, straightening up the curved crests. Computed and observed wave periods fit fairly well. The leading wavelength is in the range $10 h-25 h$.

The forward speed has a pronounced effect on the wave height, for a large depth change of $\Delta h / h \sim 1$, where $H / h$ grows according to $F r^{n}$ with $n$ in the range $3-4$. This growth with $F r$ is only very weak when $\Delta h / h<<1$ (the asymptotics). A computed wave height of up to $0.75 \mathrm{~m}(\mathrm{Fr}=0.7)$ compares to a measured wave height of $1 \mathrm{~m}$ (figure 12).

Acknowledgement. A preliminary version of this material was presented at the 31st International Workshop on Water Waves and Floating Bodies, Plymouth, Michigan, USA, 3-6 April 2016. Because of the present analysis, Color Line have reduced the speed and modified the track of their ships, strongly reducing the waves at Flaskebekk. The author is grateful to the comments and suggestions by three anonymous referees which helped improve the manuscript.

\section{Appendix A. Appendix}

\section{A.1. Further elaboration of the observations}

Flaskebekk. A wave height of $1.4 \mathrm{~m}$ was measured in the harbor at Flaskebekk, by the owner, E. Staff, using a folding rule. The harbor appears on the photographs in figures 1 and 12. A wave height of approximately $1 \mathrm{~m}$ is documented in figure 12 as the difference between the maximum and minimum elevation, corresponding to approximately 6 steps, each $17 \mathrm{~cm}$ high, of the staircase shown in the figure. The photos in figures 1 and 12 are in the same sequence, taken on the same day.

Regarding erosion, the chain of bath houses and piers along the shore line at Flaskebekk are built on unconsolidated sediments with some fractions of rock. Particularly the long run-outs lasting for approximately 15-20 seconds (half wave period) contribute to a seaward transport of the sediments, an erosion that started with the introduction of the large cruiseferries of Color Line in 2004, where the ship characteristics are given in table 4. This information is according to a group of landlords at Flaskebekk (J. Bjerke, T.H. Larsen, T. Scavenius, E. Staff). The group has told the author how they each year have to supply a significant volume of stones to the sea bottom ahead of their own bath house, to compensate for the erosion caused by the new wave environment. Those owners not making such measures experience that their bath houses are steadily tilting towards the sea. The group of landlords report that the long periodic wave run-up/run-down contribute much more to the erosion compared to the shorter wash waves of the ship wake. The pier shown in figures 1,12 is in a steady, slow seaward motion due to the erosion. The owner of Kavringen Nesodden Marina south of Flaskebekk, P. Støp, has reported to the author that the long waves contribute to a slow oscillating current in the shallow marina where the entire system of floating docks and moored boats are exposed to large excursions. This has become a new wear and tear, where the particular damage is caused by the long-lasting run-out. Wave heights in the marina have not been measured.

Askholmene. In the bay at Askholmene, estimates of the wave periods, run-up and run-down are obtained from the amateur video available at https: / www.youtube.com/watch? $\mathrm{v}=42 \mathrm{C}$ where the waves caused by Color Magic are recorded. The usual ship speed at the position is 20 knots. The run-up/run-down elevation is approximately $\pm 0.75 \mathrm{~m}$. The horizontal 
Tonnage $(\mathrm{GT}) \quad V_{0}\left(\mathrm{~m}^{3}\right) \quad l(\mathrm{~m}) \quad w_{0}(\mathrm{~m}) \quad d_{0}(\mathrm{~m}) \quad U$ (knots) Year

\begin{tabular}{|c|ccc|c|c|cc|}
\hline Magic (Color Line) & 75100 & $|36000| 224 \mid$ & 35 & 6.8 & 22 & $|2007|$ \\
\hline Fantasy (Color Line) $\mid$ & 75027 & $|36000| 224 \mid$ & 35 & 6.8 & 22 & $|2004|$ \\
\hline Pearl Seaways (DFDS) | & 40039 & $|24700| 178 \mid$ & 34 & 6.2 & 21 & $|2001|$ \\
\hline Crown Seaways (DFDS) | & 35498 & $|20200| 171 \mid$ & 28 & 6.4 & 21.5 & $|1994|$ \\
\hline Stena Saga (Stena Line) | & 33575 & $|20500| 166 \mid$ & 28 & 6.7 & 22 & $|1994|$
\end{tabular}

TABLE 4. Ship characteristics and history. Tonnage (GT), displaced volume $V_{0}\left(\mathrm{~m}^{3}\right)$, total length $(l)$, width $\left(w_{0}\right)$, draught $\left(d_{0}\right)$, maximum speed $(U)$, year in service (Year).

current velocity in the bay estimated from the video is up to $1 \mathrm{~ms}^{-1}$. The author visited the position on 19 June 2013, at the time of the day when Color Magic was cruising by. No elevation measurements were taken.

Characteristics of the five largest cruiseferries and starting year of operation in the Oslofjord are listed in table 4. The information is acquired from what is available on the Internet/Wikipedia. The displaced volume, $V_{0}$, column three in the table, is calculated using an estimated ship length between perpendiculars $\left(l_{0}\right)$, width, draugth, and a block coefficient of $C_{B}=0.7$ (see e.g. Newman, 1977, p. 350), obtaining $V_{0}=l_{0} \cdot w \cdot d \cdot 0.7$. where $l_{0}$ is put to $94 \%$ of the total length $l$.

\section{A.2. Integral equation for a small bottom step}

We consider the integral equation (3.3) in the special case when the bottom has a small step of height $\Delta h<<h$ at $x_{1}=0$ such that $y_{B}=-h$ for $x_{1}<0$ and $y_{B}=-h+\Delta h$ for $x_{1}>0$. Then $\partial / \partial n^{\prime}=\partial / \partial x_{1}^{\prime}$ at the step and $\partial / \partial n^{\prime}=-\partial / \partial y^{\prime}$ otherwise along the bottom. Using that $\partial \phi / \partial n=0$ along the bottom, the integral equation (3.3) becomes

$$
\begin{aligned}
& \iint\left(\frac{1}{R}+\frac{1}{\left(R^{2}+(2 h)^{2}\right)^{1 / 2}}\right) V^{\prime} d \mathbf{x}^{\prime}=2 \pi \phi_{F}(\mathbf{x})+\iint \phi_{F}^{\prime} \frac{\partial}{\partial(2 h)} \frac{1}{\left(R^{2}+(2 h)^{2}\right)^{1 / 2}} d \mathbf{x}^{\prime} \\
& +\left.\int_{0}^{\Delta h} \int_{-\infty}^{\infty} \phi_{B}^{\prime}\right|_{x_{1}=0} \frac{\partial}{\partial x_{1}^{\prime}}\left(\frac{1}{\left(R^{2}+\left(h-y^{\prime}\right)^{2}\right)^{1 / 2}}+\frac{1}{\left(R^{2}+\left(h+y^{\prime}\right)^{2}\right)^{1 / 2}}\right) d x_{2}^{\prime} d y^{\prime} \\
& +\int_{0}^{\infty} \int_{-\infty}^{\infty} \phi_{B}^{\prime} \frac{\partial}{\partial h}\left(\frac{1}{\left(R^{2}+(h+\Delta h)^{2}\right)^{1 / 2}}-\frac{1}{\left(R^{2}+(h-\Delta h)^{2}\right)^{1 / 2}}\right) d x_{1}^{\prime} d x_{2}^{\prime}
\end{aligned}
$$

where the two former integrals are integrated over the horizontal plane. Symbols are defined in the text above and below eq. (3.3). We obtain the Fourier transform of (A 1), giving

$$
\begin{aligned}
& \left(1+e^{-2 k h}\right) \frac{\hat{V}}{k}=\left(1-e^{-2 k h}\right) \hat{\phi}_{F}-\left.\frac{2 \mathrm{i} k_{1} e^{-k h}}{k} \int_{0}^{\Delta h} \int_{-\infty}^{\infty} \phi_{B}\right|_{x_{1}=0} \cosh \left(k y^{\prime}\right) e^{-\mathrm{i} k_{2} x_{2}^{\prime}} d x_{2}^{\prime} d y^{\prime} \\
& -2 \sinh (k \Delta h) e^{-k h} \int_{0}^{\infty} \int_{-\infty}^{\infty} \phi_{B}^{\prime} e^{-\mathrm{i} \mathbf{k} \cdot \mathbf{x}^{\prime}} d x_{2}^{\prime} d x_{1}^{\prime} .
\end{aligned}
$$


In the long wave regime $(k h<<1)$ the leading order contribution to (A 2), due to the bottom, becomes

$$
\hat{V}-k \tanh (k h) \hat{\phi}_{F}=-\mathrm{i} k_{1} \Delta h \int_{-\infty}^{\infty} \phi_{B}\left(x_{1}=0, x_{2}\right) d x_{2}+O\left(k^{2}\right),
$$

where $\hat{\phi}_{B}$ may be replaced by $\hat{\phi}_{F}$ for $k h<<1$. Eq. (A 3) expresses the dominant contribution to the vertical velocity at the free surface, for long waves, in terms of the bottom potential at the step. If the bottom transition has a finite slope the contributions from the normal derivative of the Green function become, at the slope, $\Delta S\left(\partial / \partial n^{\prime}\right)(1 / r+$ $\left.1 / r_{1}\right)=\Delta h\left(\partial / \partial x^{\prime}\right)\left(1 / r+1 / r_{1}\right)-\sqrt{(\Delta S)^{2}-(\Delta h)^{2}}\left(\partial / \partial y^{\prime}\right)\left(1 / r+1 / r_{1}\right)$, giving (A 3) as result still.

\section{A.3. Calculation of $\phi_{B}$}

The potential $\phi_{B}$ along the bottom is obtained from (3.4-3.8) giving

$$
\begin{aligned}
& 0=\hat{\Psi}+\mathcal{F}\left(\beta \mathcal{F}^{-1}\left(k \hat{\Psi}+\hat{A}_{1}+\frac{1}{2}\left(1-e_{1}^{2}\right) k \hat{A}_{2}\right)\right)+\frac{1}{2} \mathcal{F}\left(\beta^{2} \mathcal{F}^{-1}\left(k^{2} \hat{\Psi}+k^{2} \hat{\phi}_{B}\right)\right)-\frac{1}{2} e_{1}^{2} \hat{A}_{2}, \\
& +\frac{1}{6} \mathcal{F}\left(\beta^{3} \mathcal{F}^{-1}\left(k^{3} \hat{\Psi}+k^{2}\left(1+e_{1}^{2}\right) \hat{A}_{1}\right)+\ldots\right),
\end{aligned}
$$

where the variable $\Psi$ is defined by

$$
k \hat{\Psi}=-k \hat{\phi}_{B}+2 \hat{V} e_{1}-\left(1+e_{1}^{2}\right) \hat{A}_{1}-\frac{1}{6}\left(1+e_{1}^{2}\right) k^{2} \hat{A}_{3},
$$

and where $T_{1}, C_{1}$ and $\hat{A}_{n}$ are defined below (3.10), and $e_{1}=e^{-k h}$. Note that the leading two approximands of $\Psi$ in $(\mathrm{A} 5)$ are zero, i.e. $\hat{\Psi}^{(0)}=\hat{\Psi}^{(1)}=0$ where the order $n$ refers to terms proportional to $\beta^{n}$. The functions $\hat{\Psi}^{(2)}$ and $\hat{\phi}_{B}^{(2)}$ are obtained iteratively from (A 4-(A 5) keeping terms proportional $\beta^{2}$, while $\hat{\Psi}^{(2)}+\hat{\Psi}^{(3)}$ and $\hat{\phi}_{B}^{(2)}+\hat{\phi}_{B}^{(3)}$ are obtained iteratively from (A 4-A 5) keeping terms proportional to both $\beta^{2}$ and $\beta^{3}$.

\section{References}

Brown, E.D., Bucksbaum, S.B., Hall, R.E., Penhune, J.P., Schmitt, K.F., Watson, K.M. and Wyatt, D.C. (1989) Observation of a nonlinear solitary wave packet in the Kelvin wake of a ship. J. Fluid Mech., 204, 263-293.

Clamond, D. and Grue, J. (2001) A fast method for fully nonlinear water wave computations. J. Fluid Mech., 447, 337-355.

Clarisse, J.-M., Newman, J.N. and Ursell, F. (1995) Integrals with a large parameter: water waves on finite depth due to an impulse. Proc. Roy. Soc. A, 450, 67-87.

Cole, S.L. (1985) Transient waves produced by flow past a bump. Wave Motion, 7, 579587.

Constantine, T. (1960) On the movement of ships in restricted waterways. J. Fluid Mech., 9, 247-256.

Didenkulova, I., Pelinovsky, E. and Soomere, T. (2011) Can the waves generated by fast ferries be a physical model of tsunami? Pure Appl. Geophys., 168, 2071-2082.

Ertekin, R.C., Webster, W.C. and Wehausen, J.V. (1986) Waves caused by a moving disturbance in a shallow channel of finite width. J. Fluid Mech., 169, 275-292.

Fructus, D. and Grue, J. (2007) An explicit method for the nonlinear interaction between water waves and variable and moving bottom topography. J. Comp. Phys., 222, 720-739. Glimsdal, S., Pedersen, G.K., Atakan, K., Harbitz, C.B., Langtangen, H.P. and Lovholt, 
F. (2006) Propagation of the Dec. 26, 2004, Indian Ocean Tsunami. Effects of dispersion and source characteristics. Int. J. Fluid Mech. Res. 33, 15-43.

Grue, J. (2002) On four highly nonlinear phenomena in wave theory and marine hydrodynamics. Appl. Ocean Res., 24, 261-274.

Grue, J. (2015) Nonlinear interfacial wave formation in three dimensions. J. Fluid Mech., $767,735-762$.

Hamer, M. (1999) Solitary killers. New Scientist 163(2291), 18-19.

Harbitz, C.B. (1992) Model simulations of tsunamis generated by the Storegga slides. Marine Geology 105, 1-21.

Mei, C.C. (1989) The applied dynamics of ocean surface waves. World Scientific. 740 pp. Newman, J.N. (1977) Marine hydrodynamics. MIT Press. 402 pp.

Pedersen, G. (1988) Three-dimensional wave patterns generated by moving disturbances at transcritical speeds. J. Fluid Mech., 196, 39-63.

Pelinovksy, E. (2006) Hydrodynamics of tsunami waves. In: J. Grue and K. Trulsen (Eds.) Waves in Geophysical Fluids, CISM Courses and Lectures No. 489, Springer, 2006, pp. $1-48$.

Sibul, O.J., Webster, W.C. and Wehausen, J.V. (1979) B. A phenomenon observed in transient testing. Schiffstechnik, 26, 179-200.

Soomere, T. (2005) Fast ferry traffic as a qualitatively new forcing factor of environmental processes in non-tidal sea areas: A case study in Tallinn Bay, Baltic Sea. Environm. Fluid Mechanics 5, 293-323.

Soomere, T. (2009) Long ship waves in shallow water bodies. In E. Quak and T. Soomere (Eds.) Applied Waves Mathematics. Springer-Verlag. pp. 193-228.

Torsvik, T. (2009) Modelling of ship waves from high-speed vessels. In E. Quak and T. Soomere (Eds.) Applied Waves Mathematics. Springer-Verlag. pp. 229-263.

Torsvik, T., Pedersen, G. and Dysthe, K. (2009) Waves generated by a pressure disturbance moving in a channel with a variable cross-sectional topography. J. Waterway, Port, Coastal and Ocean Eng., 135(5), 120-123.

Wu, J. and Chen, B. (2003) Unsteady ship waves in shallow water of varying depth based on Green-Naghdi equation. Ocean engineering, 30, 1899-1913.

Wu, T.Y. (1987) Generation of upstream advancing solitons by moving disturbances. J. Fluid Mech., 184, 75-99. 


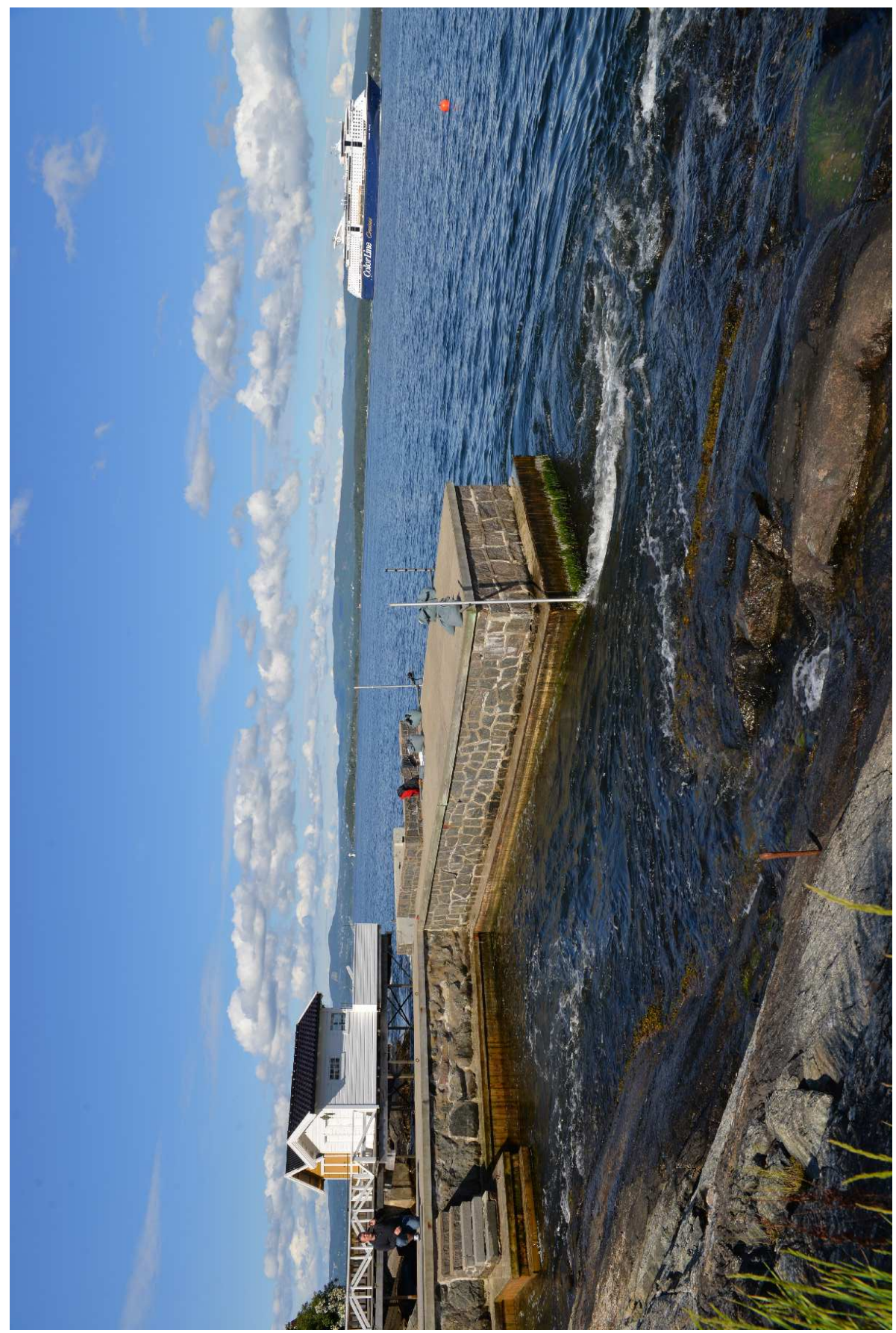

FIGURE 1. Outflow of tsunami in harbor at Flaskebekk, generated by the ship Color Magic seen in the picture. The generation site in the fjord is next to the lighthouse seen above the pier and to the right of the white bathhouse. The distance to the ship is approximately $1.2 \mathrm{~km}$. Photo. T.H. Larsen. 
a)

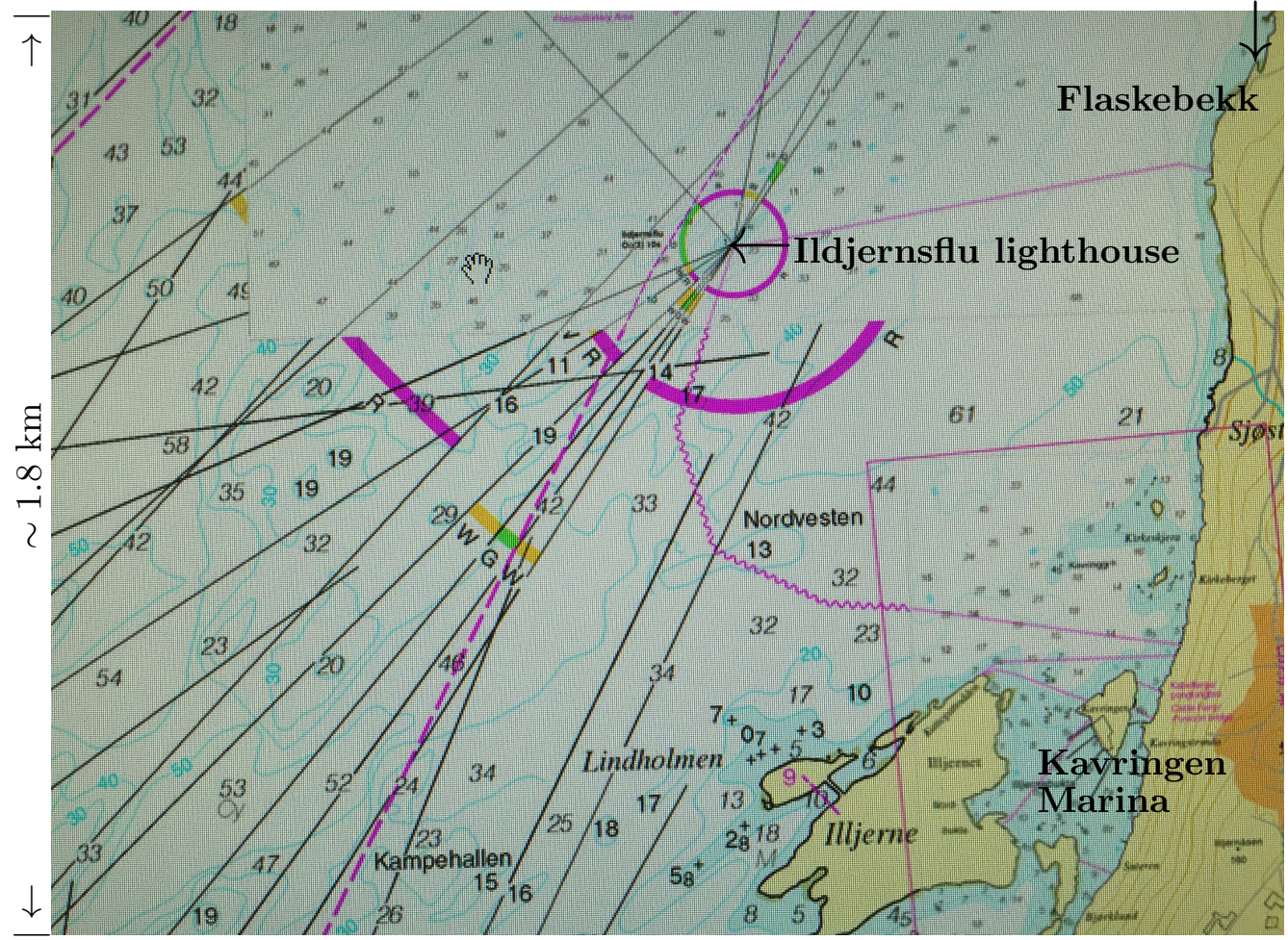

b)

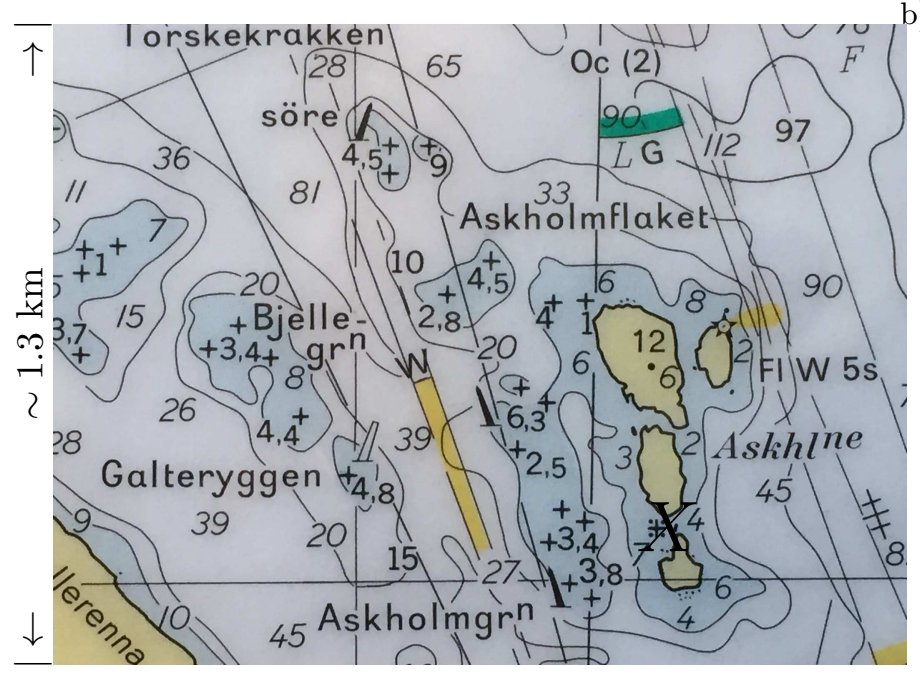

c)

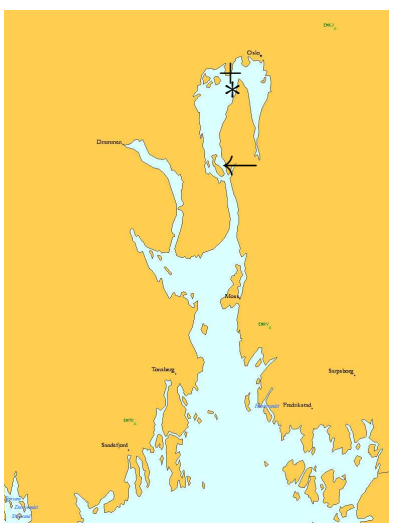

Figure 2. a) Section of seachart of the Oslofjord at Ildjernsflu, with marked position of the Ildjernsflu lighthouse $(\leftarrow)$ and the harbor at Flaskebekk, imaged in figure $1(\downarrow)$. The ship track is to the left of the violet dashed line. b) Seachart at Askholmene with position of videorecording marked by $X$. The ship track crosses over depths of $81 \mathrm{~m}, 39 \mathrm{~m}, 27 \mathrm{~m}$. c) Overview with positions of Ildjernsflu/Flaskebekk $(*)$, Askholmene $(\leftarrow)$ and Snarøya $(+)$ indicated. a-b) are minor sections of charts available at www.oslofjorden.com/map/. The Oslofjord is $109 \mathrm{~km}$ long. 

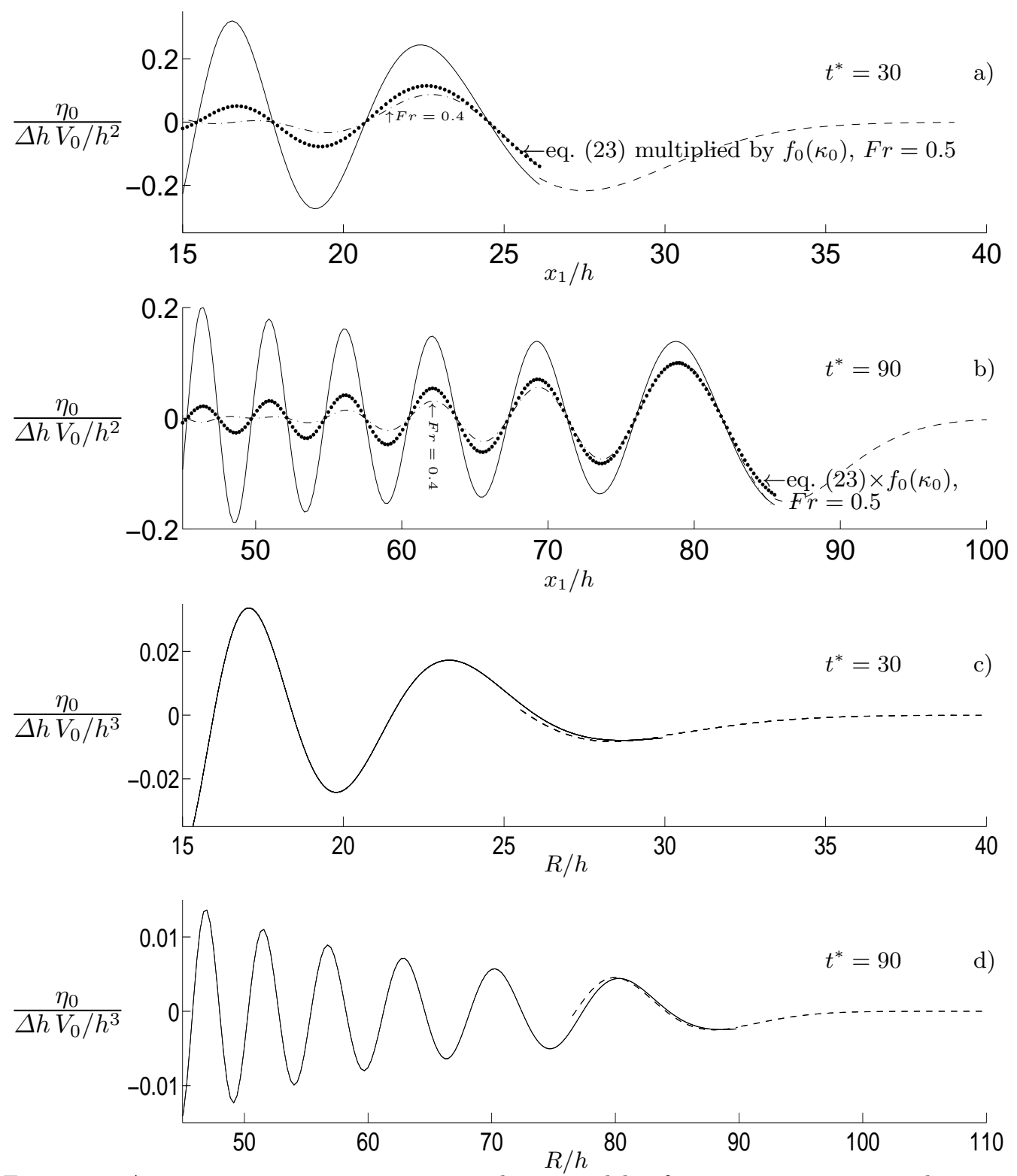

Figure 3. Asymptotic upstream wave train due to a delta function moving over a bottom step with $\Delta h>0$ for $F r=0.5$ and $t^{*}=t \sqrt{g / h}=30,90$. a)-b) Two-dimensional case obtained by (4.6) (- ), leading part of elevation obtained by (4.7) $(---)$, (4.6) multiplied by $f_{0}\left(\kappa_{0}\right)=8 J_{2}\left(\kappa_{0}\right) / \kappa_{0}^{2}, \kappa_{0}=\omega_{0} l_{0} /(2 U), l_{0} / h=3.82$, for $F r=0.5$ (thick dots) and $F r=0.4$ $(-\cdot-\cdot)$. c)-d) Three-dimensional case. Cut along the $x_{1}$-axis with $(4.11)(-)$ and $(4.12)(---)$. 


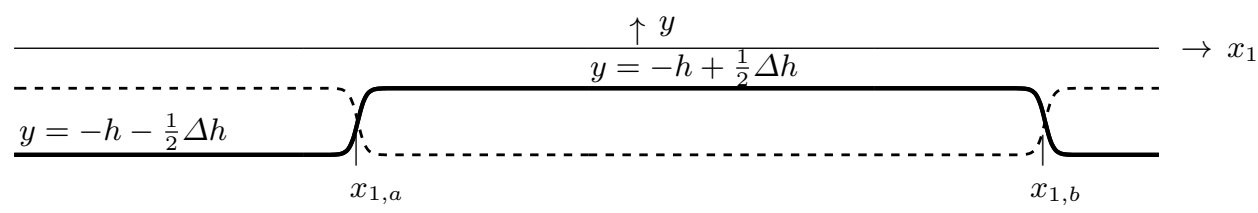

FiguRE 4 . Bottom geometries, section at $x_{2}=$ const. 

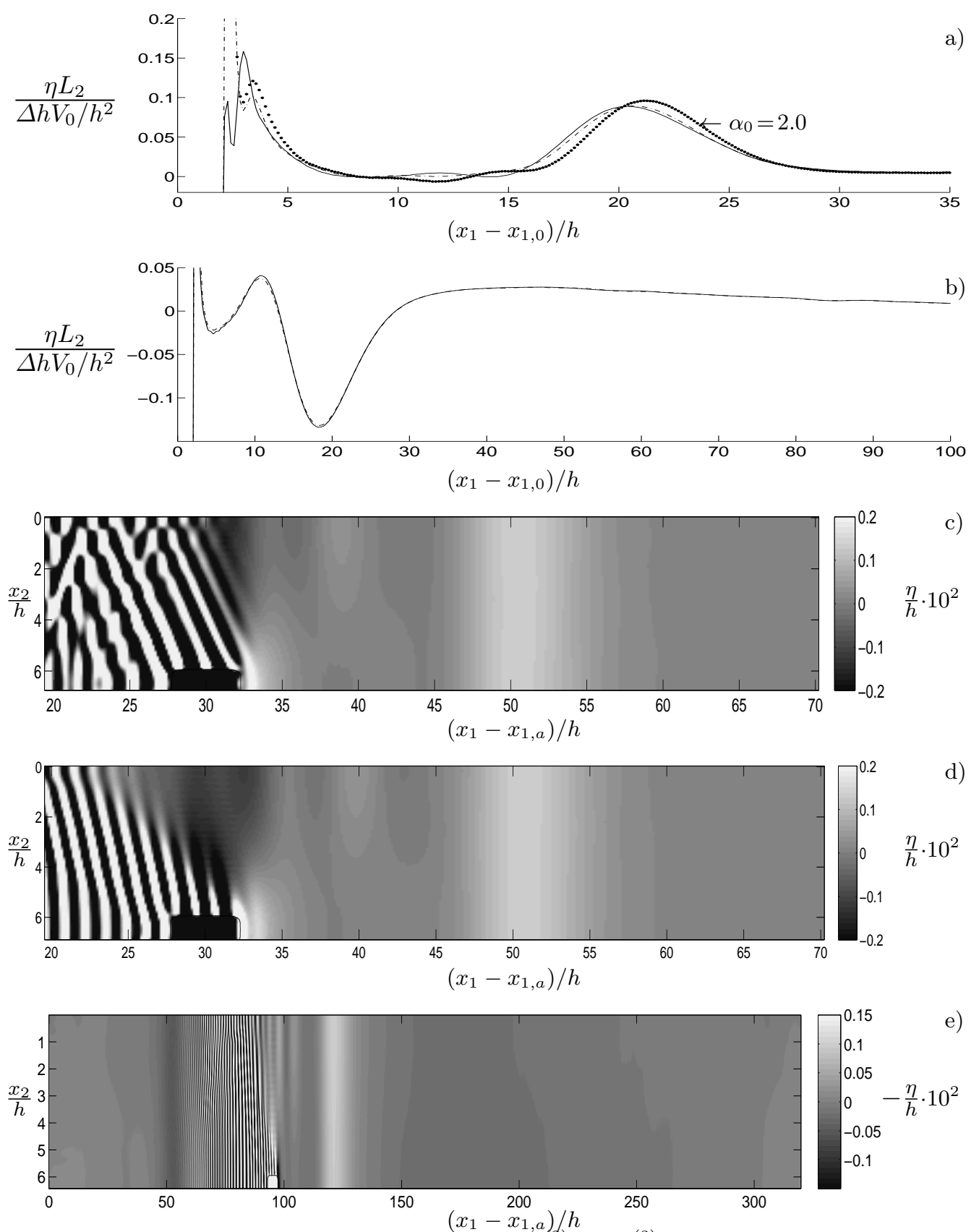

Figure 5. Elevation $\eta$ obtained by approximations $s^{(2)}$ and $s^{(3)}$ for $\Delta h / h=0.909$ and $h=55$ m. $V_{0} / h^{3}=0.216$. a) $\Delta h>0$. $\eta$ in the middle of the channel, as function of $x_{1}-x_{1,0}$, for $t^{*}=281$ with $s^{(2)}(-\cdot-\cdot), s^{(3)}(-)$ and $\alpha_{0}=0.7$ in both cases, and $s^{(2)}$ with $\alpha_{0}=2.0$ (dots). b) $\Delta h<0 . \eta$ in the middle of the channel, as function of $x_{1}-x_{1,0}$, for $t^{*}=260$ with $s^{(2)}(-\cdot-\cdot), s^{(3)}(-)$ and $\alpha_{0}=0.7$. c) $\Delta h>0 . \eta$ for $t^{*}=281$ by $s^{(3)}$. d) Same as c) but $s^{(2)}$.

e) $\Delta h<0$. Entire computational domain for $t^{*}=293$. 

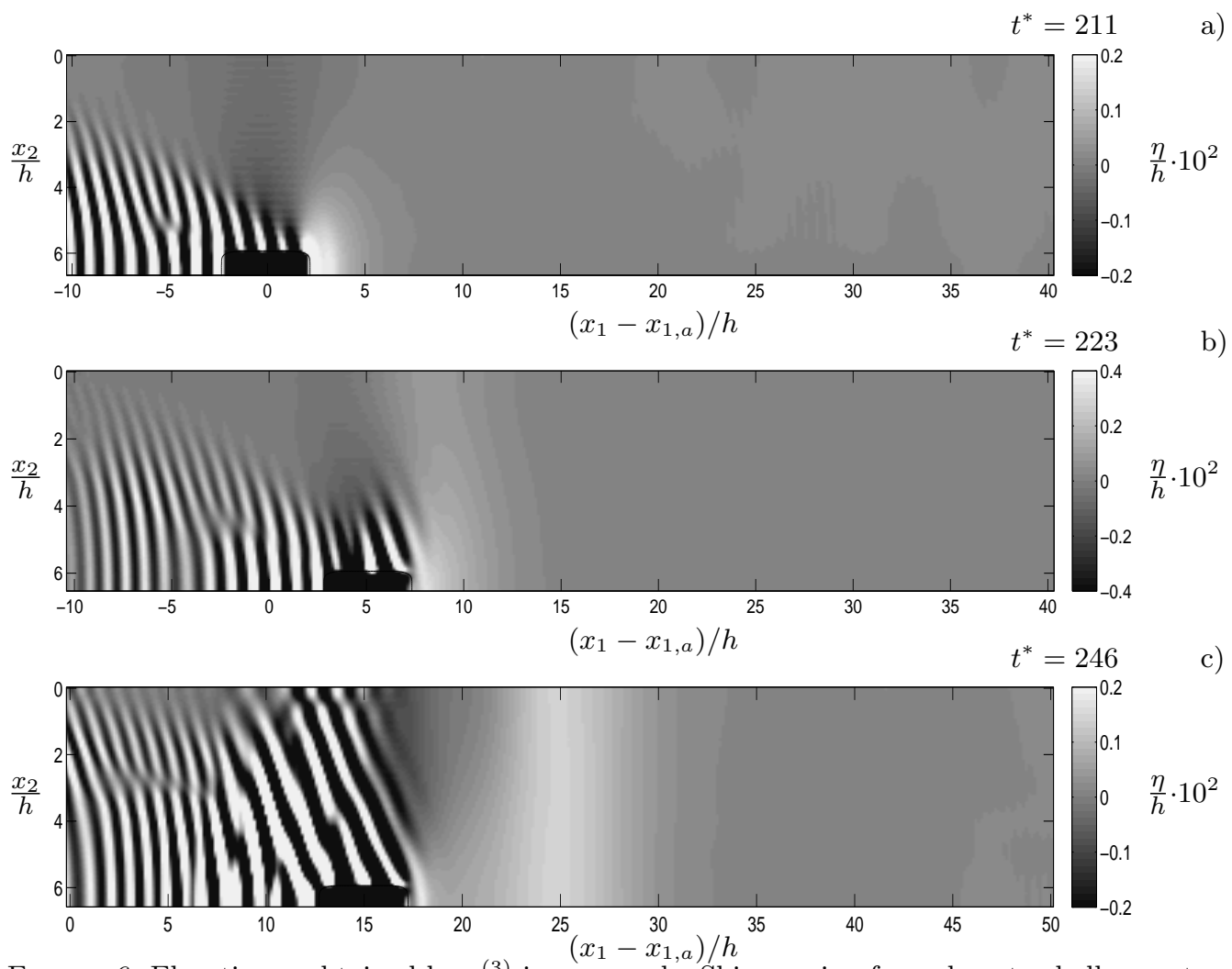

Figure 6 . Elevation $\eta$ obtained by $s^{(3)}$ in grey scale. Ship moving from deep to shallow water. $F r=0.43$. a) $t^{*}=211$, b) 223 , c) 246 . Center position of depth change at $x_{1}-x_{1, a}=0$. $\Delta h / h=0.909$. 

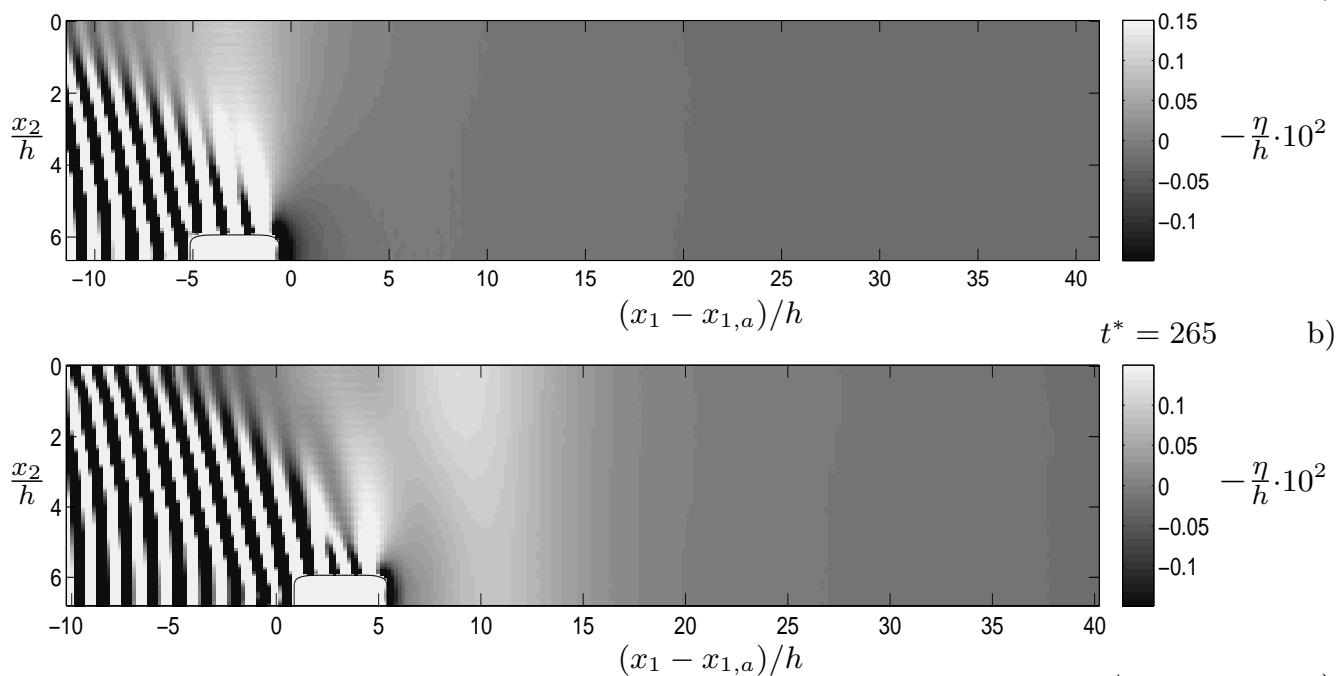

$t^{*}=265$

b)
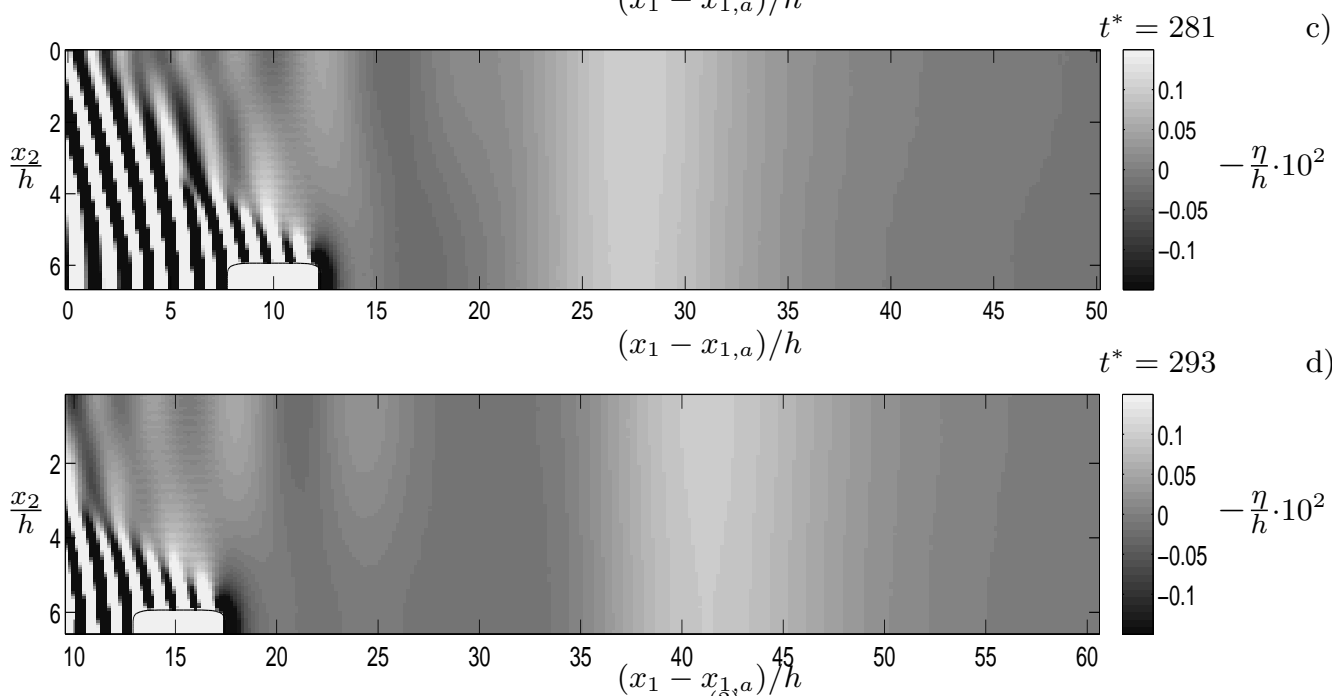

Figure 7 . The negative elevation $-\eta$ obtained by $s^{(2)}$ in grey scale. $F r=0.43$. Ship moving from shallow to deep water. Times: a) $t^{*}=251$, b) 265 , c) 281 , d) 293. Depth change at $x_{1}-x_{1, a}=0$. $\Delta h / h=0.909$. 


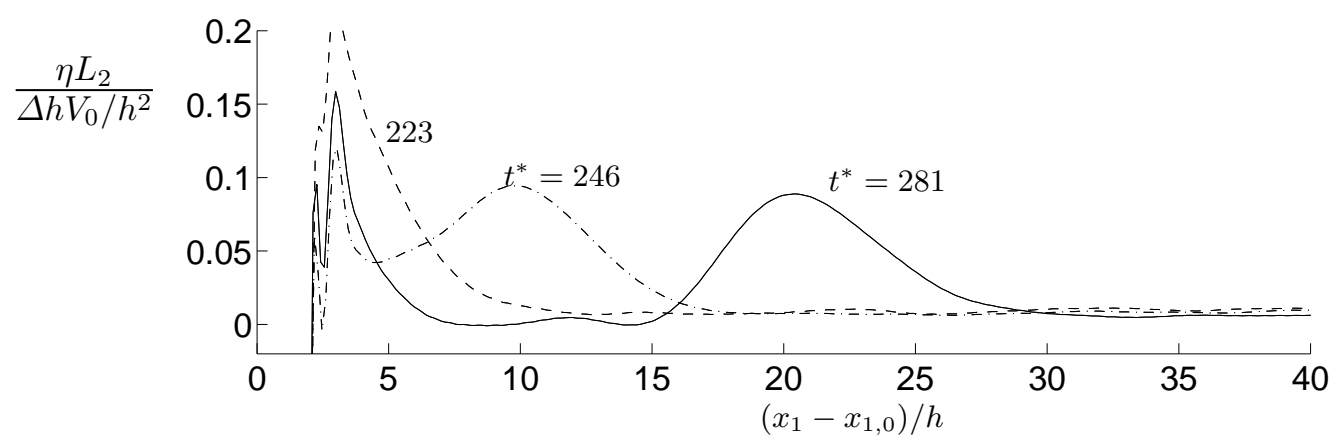

b)

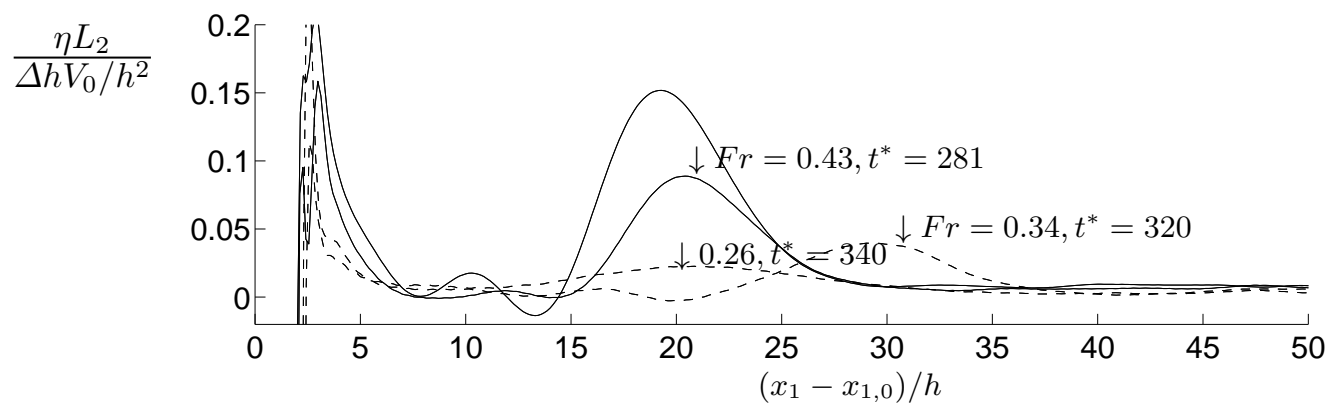

c)

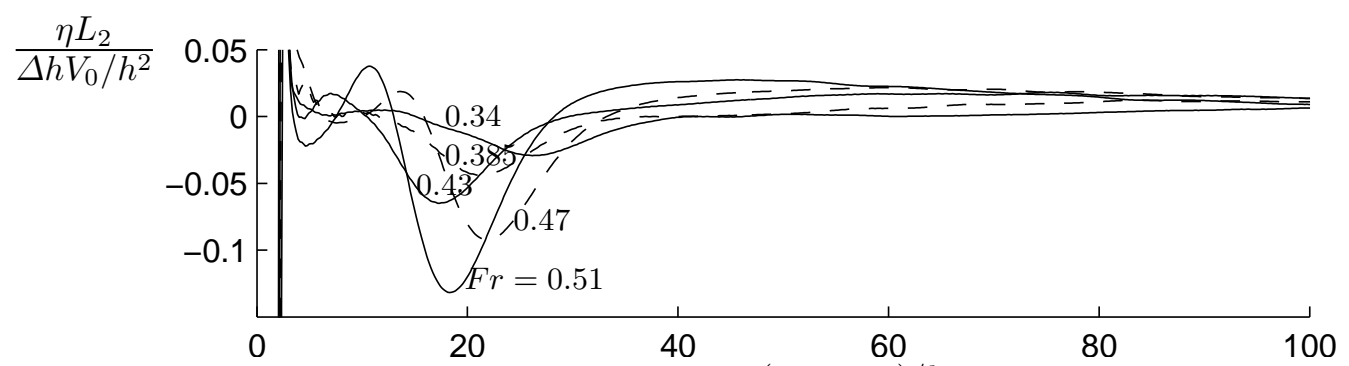

FIGURE 8. Upstream elevation $\eta L_{2} /\left(\Delta h V_{0} / h^{2}\right)$ ahead of ship. $\left(x_{1,0}\right) h / h=0.909$. a) Deep to shallow water; $F r=0.43, t^{*}=223,246,281$ calculated by $s^{(3)}$. b) $F r=0.43$ at $t^{*}=281$ and 0.51 at $t^{*}=280$ by $s^{(3)}$ (solid line); $F r=0.26$ at $t^{*}=340,0.34$ at $t^{*}=320$ by $s^{(2)}$ (dashed line). c) Ship moving from shallow to deep water. $\mathrm{Fr}=0.34,0.385,0.43,0.47,0.51$. Calculations by $s^{(2)}$. 


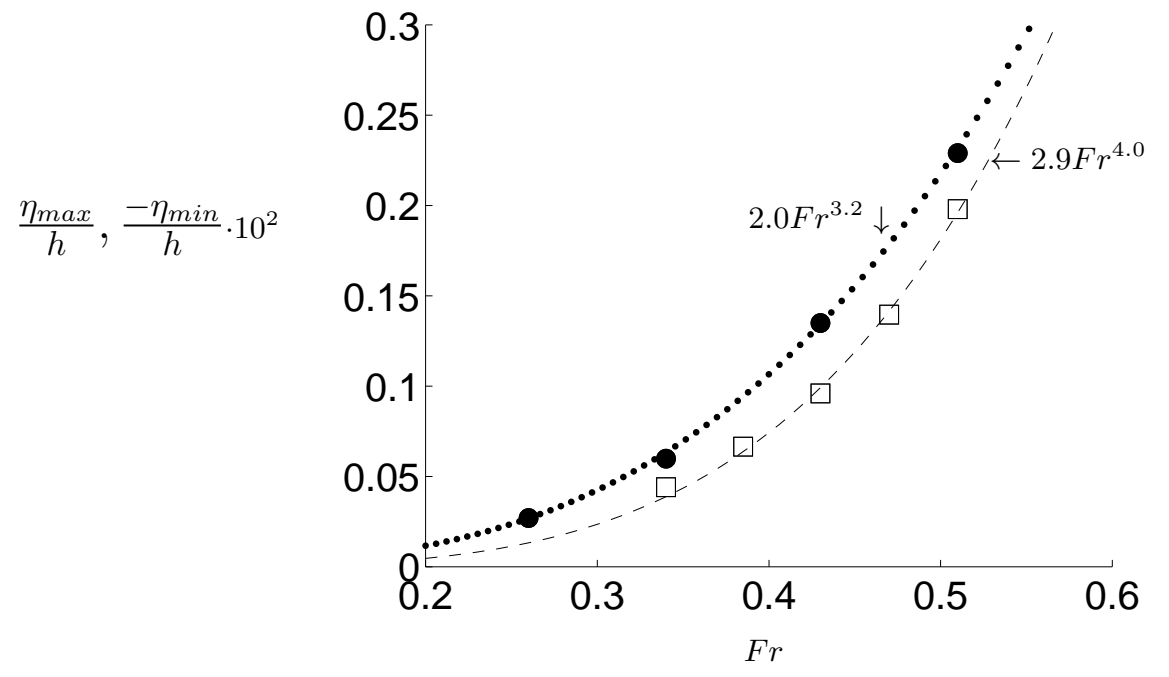

a)
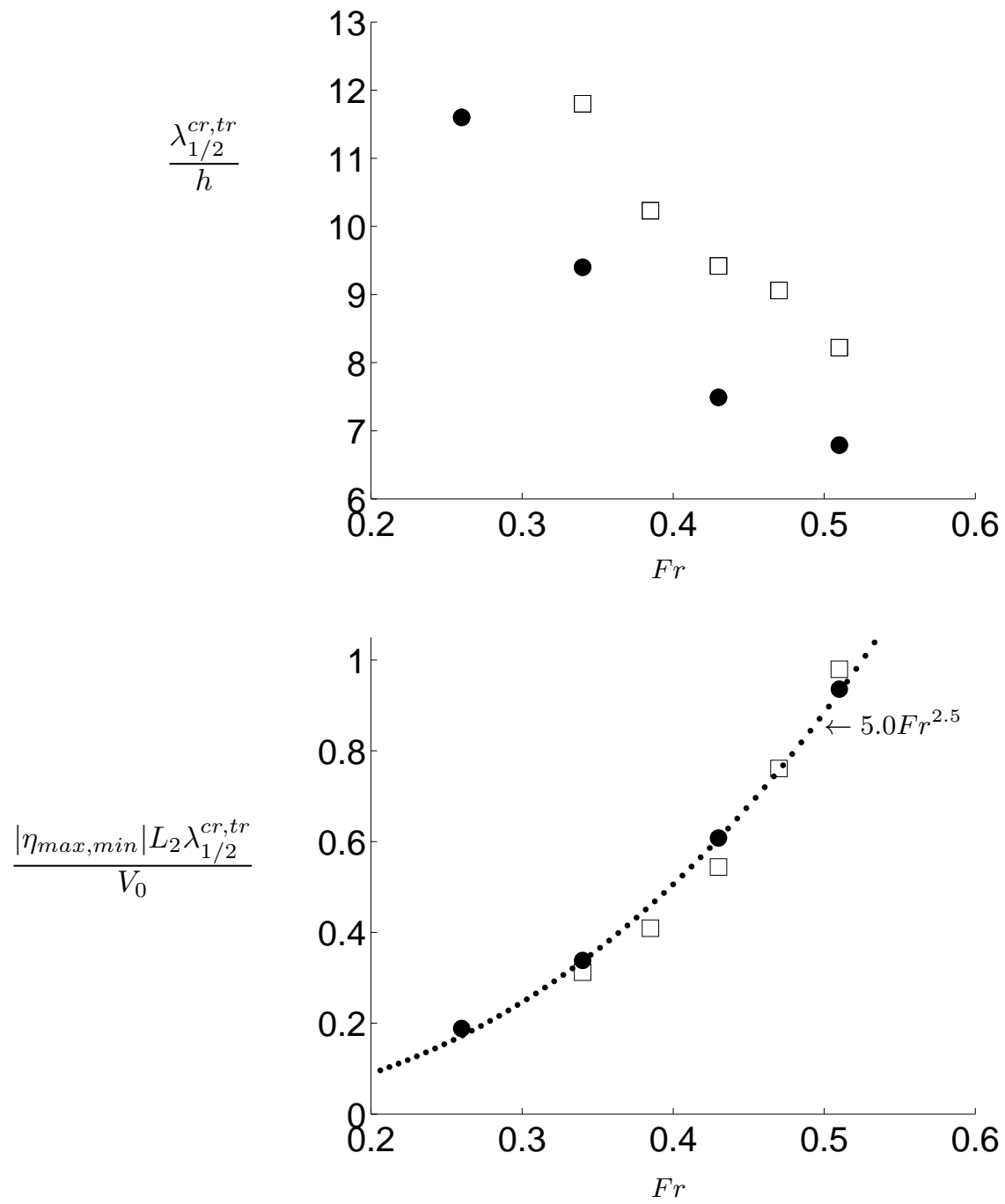

b)

c)

Figure 9. a) Maximum upstream elevation $\eta_{\max }(\bullet)$ for ship moving from deep to shallow water, and maximum upstream depression $\eta_{\min }(\square)$ for ship moving from shallow to deep water vs. $F r . \Delta h / h=0.909$. Fitted curves: dotted and dashed lines. b) $\lambda_{1 / 2}^{c r, t r} / h$ vs. Fr. c) $\eta_{\max } L_{2} \lambda_{1 / 2}^{c r} / V_{0}$ 

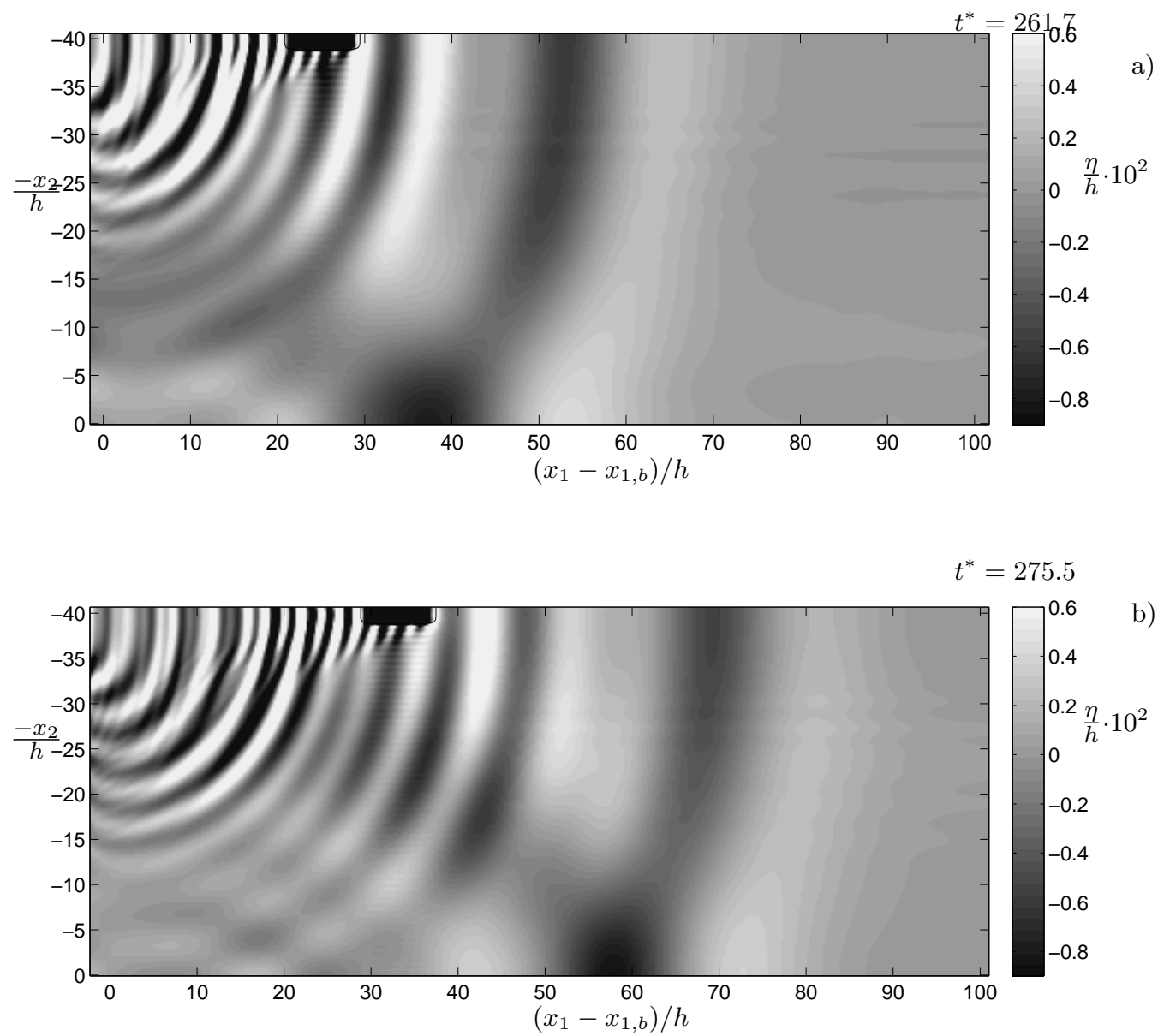

$t^{*}=311.4$

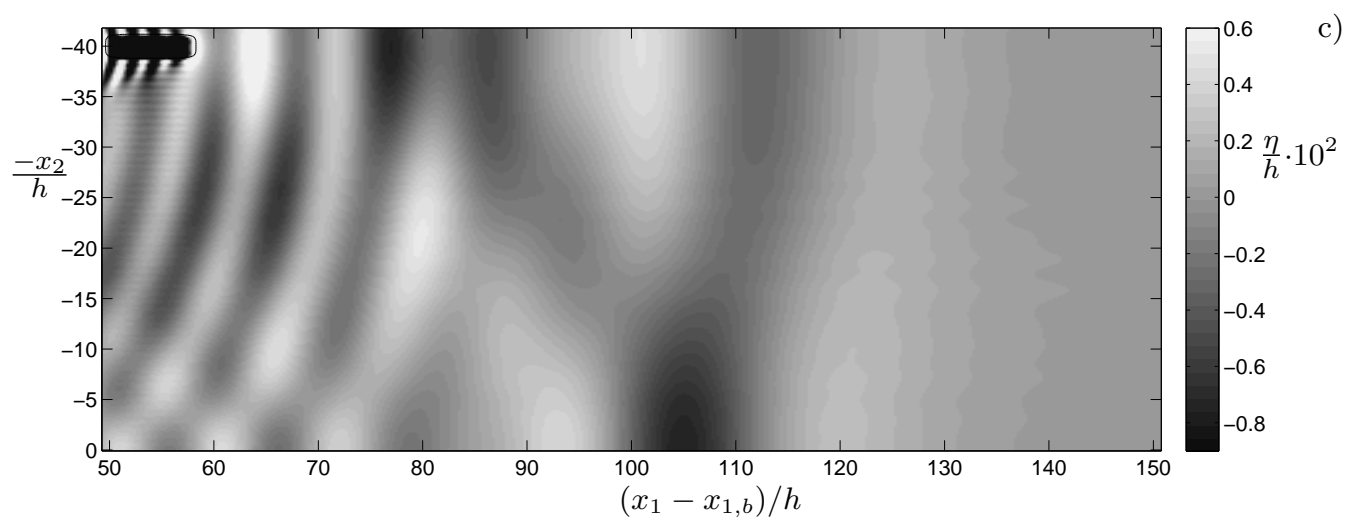

FigURE 10. a) Upstream elevation pattern obtained by $s^{(2)}$ for times a) $t^{*}=261.7$, b) 275.5 , c) 311.4. Ship with $F r=0.58$ passing from deep to shallow to deep water with $x_{1, a}=60 h$, $x_{1, b}=83 h$ and $\Delta h / h=1.067$. Horizontal coordinate $\left(x_{1}-x_{1, b}\right) / h$ with end of topography located in $x_{1}-x_{1, b}=0 . L_{1}=300 h, L_{2}=80 h, N_{1}=1200, N_{2}=432$. Depth $h=30 \mathrm{~m}$. 
a)
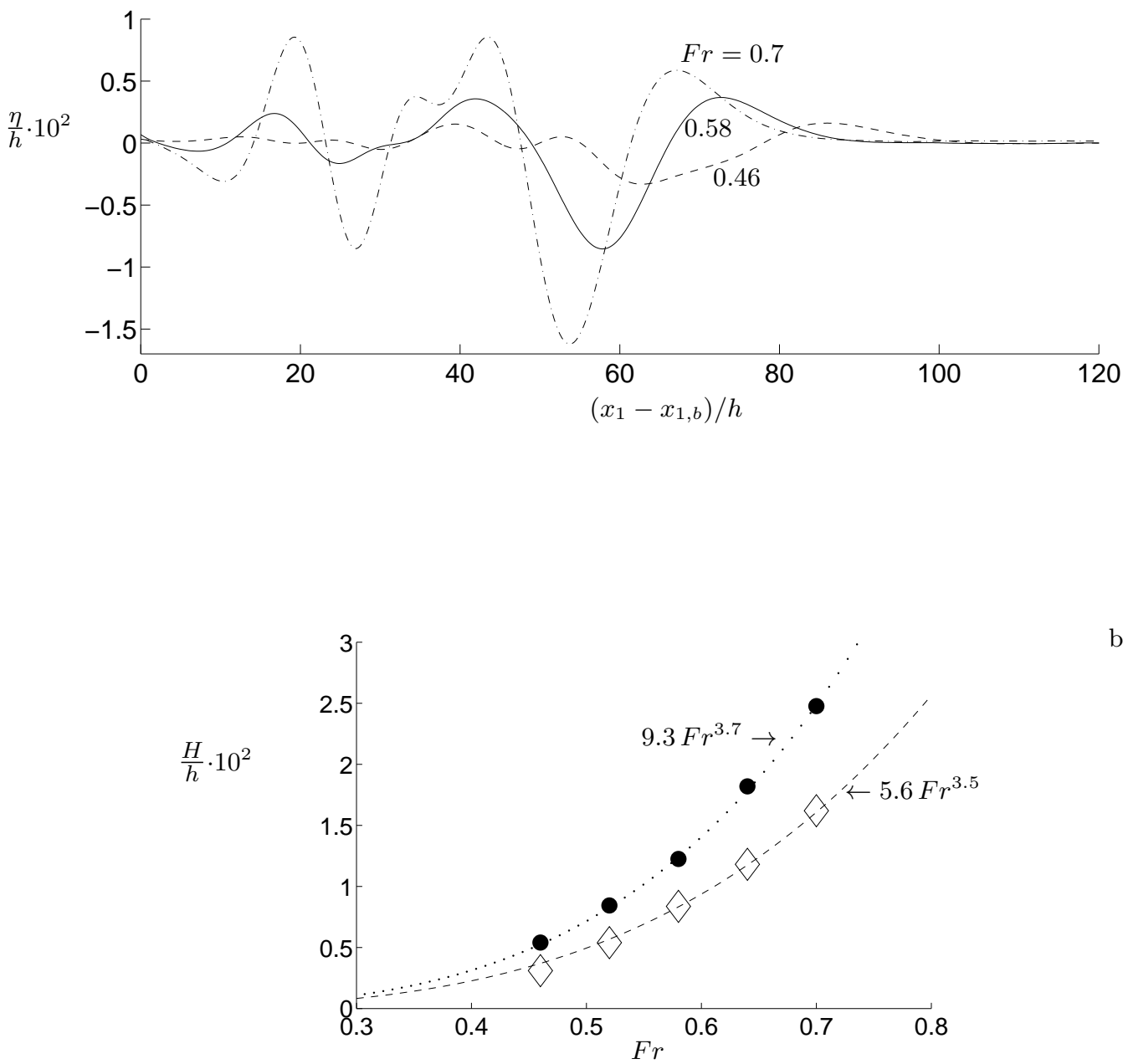

b)

FiguRE 11. a) Upstream wave elevation along channel wall obtained by $s^{(2)}$ as function of $\left(x_{1}-x_{1, b}\right) / h$ for $F r=0.7, t^{*}=252.8(-\cdot-\cdot), F r=0.58, t^{*}=287.4(-)$ and $F r=0.46$, $t^{*}=311(---)$. b) Maximum wave height at channel wall at $x_{2}=0, x_{1}-x_{1, b}=50 h(\bullet)$ and ahead of ship at $x_{2}=40 h, x_{1}-x_{1, b} \sim 60 h(\diamond)$. Fitted curves (dotted and dashed lines). $\Delta h / h=1.067, h=30 \mathrm{~m}$. Same discretization as in figure 10 . 


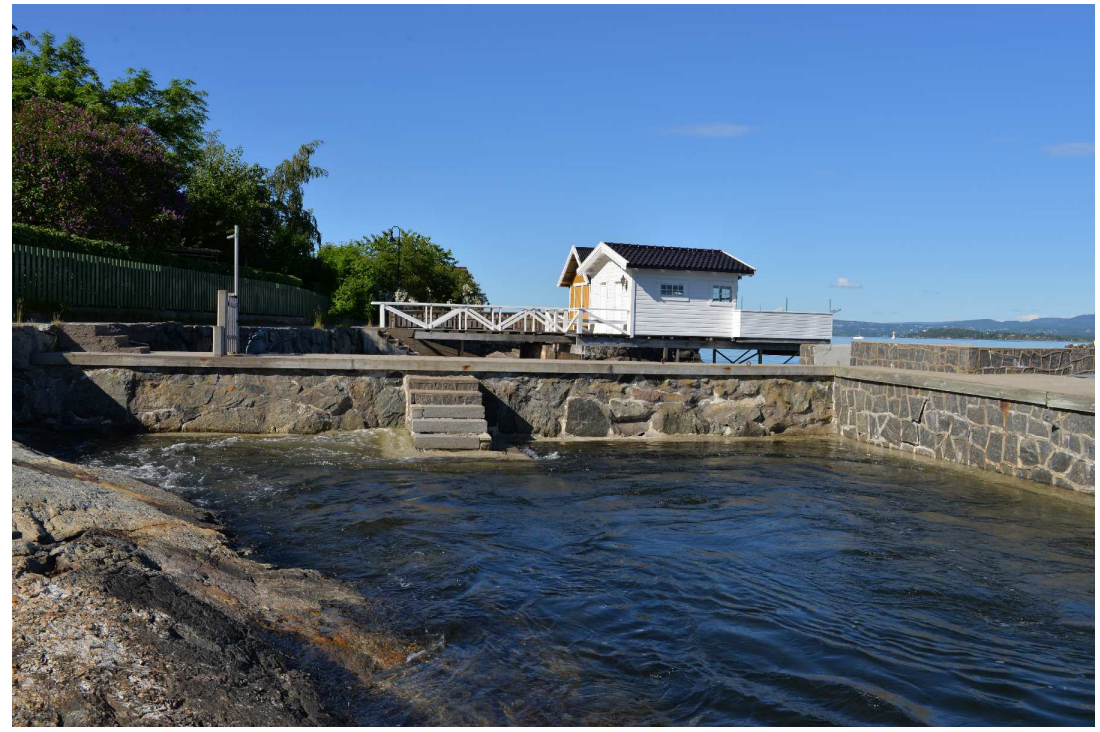

a)

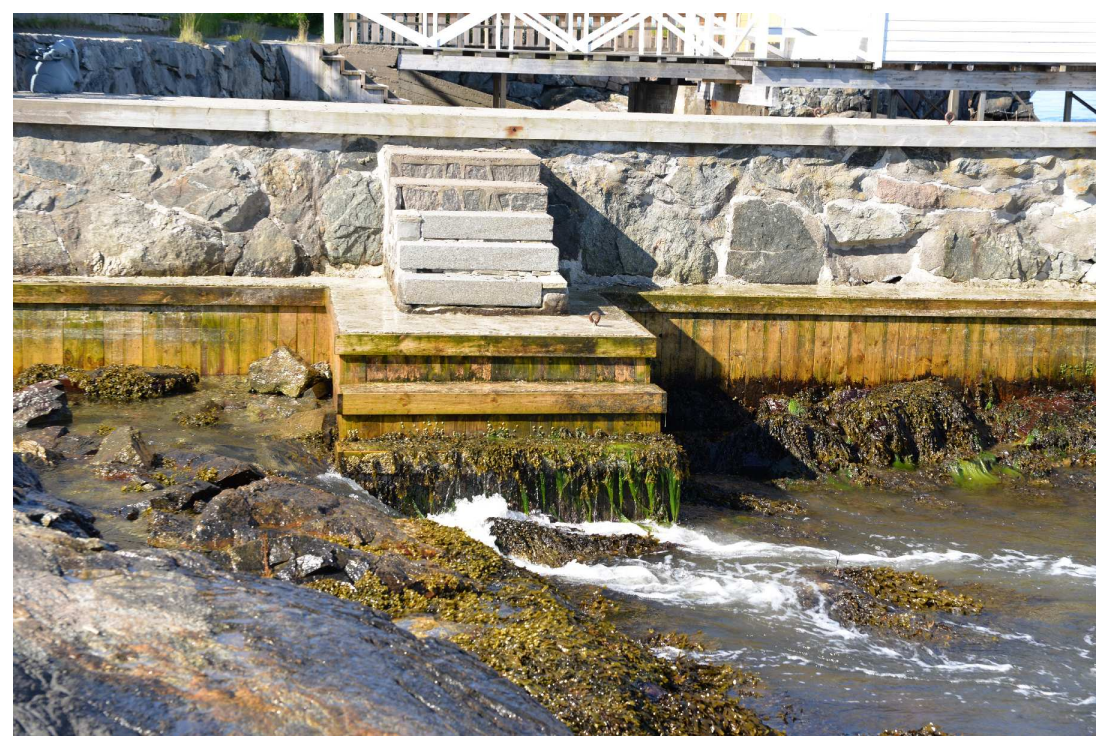

b)

Figure 12. a) Maximum and b) minimum elevation in harbor at Flaskebekk. Photo. T.H. Larsen. 